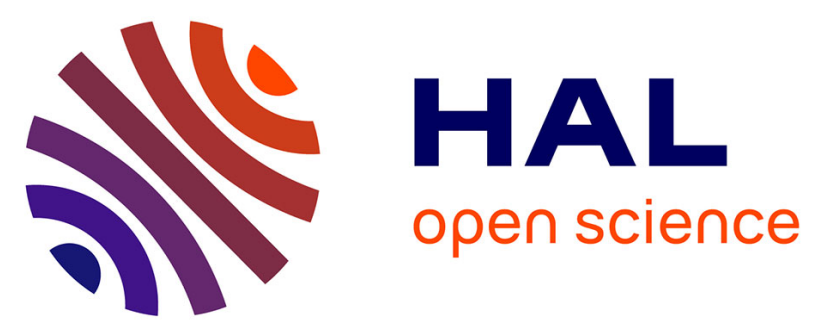

\title{
Error-related modulations of the sensorimotor post-movement and foreperiod beta-band activities arise from distinct neural substrates and do not reflect efferent signal processing
}

Julie Alayrangues, Flavie Torrecillos, Amirhossein Jahani, Nicole Malfait

\section{To cite this version:}

Julie Alayrangues, Flavie Torrecillos, Amirhossein Jahani, Nicole Malfait. Error-related modulations of the sensorimotor post-movement and foreperiod beta-band activities arise from distinct neural substrates and do not reflect efferent signal processing. NeuroImage, 2019, 184, pp.10-24. 10.1016/j.neuroimage.2018.09.013 . hal-03566554

\author{
HAL Id: hal-03566554 \\ https://hal.science/hal-03566554
}

Submitted on 11 Feb 2022

HAL is a multi-disciplinary open access archive for the deposit and dissemination of scientific research documents, whether they are published or not. The documents may come from teaching and research institutions in France or abroad, or from public or private research centers.
L'archive ouverte pluridisciplinaire HAL, est destinée au dépôt et à la diffusion de documents scientifiques de niveau recherche, publiés ou non, émanant des établissements d'enseignement et de recherche français ou étrangers, des laboratoires publics ou privés. 


\title{
Error-related modulations of the sensorimotor post-movement and foreperiod beta-band activities arise from distinct neural substrates and do not reflect efferent signal processing
}

\author{
Julie Alayrangues ${ }^{a}$, Flavie Torrecillos ${ }^{\mathrm{b}}$, Amirhossein Jahani ${ }^{\mathrm{a}}$, Nicole Malfait ${ }^{\mathrm{a}, \mathrm{*}}$ \\ ${ }^{a}$ Institut de Neurosciences de la Timone, UMR7289, Aix-Marseille Université/CNRS, Marseille, France \\ b Nuffield Department of Clinical Neurosciences, John Radcliffe Hospital, University of Oxford, Oxford, UK
}

\section{A R T I C L E I N F O}

\section{Keywords:}

EEG

Error-processing

Interlimb coordination

Beta oscillations

Sensorimotor adaptation

\begin{abstract}
A B S T R A C T
While beta activity has been extensively studied in relation to voluntary movement, its role in sensorimotor adaptation remains largely uncertain. Recently, it has been shown that the post-movement beta rebound as well as beta activity during movement-preparation are modulated by movement errors. However, there are critical functional differences between pre- and post-movement beta activities. Here, we addressed two related open questions. Do the pre- and post-movement error-related modulations arise from distinct neural substrates? Do these modulations relate to efferent signals shaping muscle-activation patterns or do they reflect integration of sensory information, intervening upstream of the motor output? For this purpose, first we exploited independent component analysis (ICA) which revealed a double dissociation suggesting that distinct neural substrates are recruited in error-related beta-power modulations observed before and after movement. Second, we compared error-related beta oscillation responses observed in two bimanual reaching tasks involving similar movements but different interlimb coordination, and in which the same mechanical perturbations induced different behavioral adaptive responses. While the task difference was not reflected in the post-movement beta rebound, the premovement beta activity was differently modulated according to the interlimb coordination. Critically, we show an uncoupling between the behavioral and the electrophysiological responses during the movement preparation phase, which demonstrates that the error-related modulation of the foreperiod beta activity does not reflect changes in the motor output from primary motor cortex. It seems instead to relate to higher level processing of sensory afferents, essential for sensorimotor adaptation.
\end{abstract}

\section{Introduction}

Modulation of human EEG beta $(15-30 \mathrm{~Hz})$ oscillations in relation to voluntary movement was reported several decades ago (Neuper and Pfurtscheller, 1996). However, the functional significance of beta activity in relation to movement-error processing and sensorimotor adaptation processes is still uncertain. Tan et al. (2014) first demonstrated that the beta rebound, an increase in beta power typically observed at the end of movement, is modulated by kinematic errors. Using a hand-controlled-joystick task, they observed that the beta rebound was attenuated for movements in which movement-execution errors were induced by a visual perturbation. Moreover, they demonstrated that this effect was stronger when the context enhanced the salience of the kinematic errors and proposed that the beta rebound signals neuronal activity implementing Bayesian inference to update internal models during sensorimotor adaptation (Tan et al., 2014). More precisely, it would index the estimation uncertainty, inherent to the forward internal model, about the sensory consequences of the motor-command (Tan et al., 2016).

In a whole-arm reaching task, together with the beta rebound at the end of perturbed trials, we examined beta activity during the preparation (foreperiod) of reaches directly following a perturbed trial (Torrecillos et al., 2015). In contrast to the gradual decrease in beta power immediately preceding movement initiation, which has been extensively described (Nagamine et al., 1996; Pfurtscheller and Lopes da Silva, 1999; Taniguchi et al., 2000; Paradiso et al., 2004), only a limited number of

\footnotetext{
* Corresponding author. Aix-Marseille Université/CNRS, UMR7289, Institut de Neurosciences de la Timone (INT), Campus santé Timone, 27, boulevard Jean Moulin, 13385, Marseille cedex 5, France.

E-mail address: nicole.malfait@univ-amu.fr (N. Malfait).
} 
studies have examined beta activity further back in time during the foreperiod (for a review see Kilavik et al., 2013). However, several studies using pre-cueing paradigms have reported intermittent beta peaks during the foreperiod between the warning and the imperative cues (Alegre et al., 2004, 2006; Molnár et al., 2008; Fischer et al., 2010; Van Wijk et al., 2009; Zaepffel et al., 2013), and suggested a link between beta oscillations and motor planning in the foreperiod (for a review, see Kilavik et al., 2013). In our previous study (Torrecillos et al., 2015) we contrasted oscillatory responses evoked by two types of reach errors: errors that trigger trial-to-trial motor-command update and errors that do not elicit sensorimotor adaptation. We found that the post-movement beta rebound was similarly attenuated for both types of errors, whereas in contrast the foreperiod beta activity was sensitive to the nature of the perturbation. Specifically, the transient beta power enhancement, peaking around $1 \mathrm{~s}$ before movement onset, was attenuated before reaches that followed a movement-execution error activating sensorimotor adaptation, but not after a perturbation that did not trigger trial-to-trial motor-command update. On the basis of these distinct patterns, we proposed that the attenuation of the beta rebound reflects error-salience processing independent of sensorimotor adaptation. In contrast, the modulation of the foreperiod beta power seems to relate to adaptive processes activated after a movement-execution error is experienced.

While they uncover critical functional differences, these findings leave unresolved two central and related issues. First, it remains unknown whether the same or different neural substrates are involved in the pre- and post-movement error-related oscillatory responses. In general, power modulations in brain oscillations may be related to the degree of spike synchronization (Denker et al., 2011) and/or the overall level of activity in neuronal populations (Nauhaus et al., 2009). It has been shown that beta oscillations can synchronize over large networks, spanning multiple cortical (Brovelli et al., 2004; Murthy and Fetz, 1992, 1996; Roelfsema et al., 1997) and sub-cortical (Courtemanche and Lamarre, 2005; Courtemanche et al., 2003) areas. Therefore sensorimotor beta power modulations may reflect different neuronal populations and/or network states. Second, our previous findings do not provide any insight into the nature of the adaptive processes that are reflected by the foreperiod beta modulation. Traditionally sensorimotor beta activity has been considered in relation to descending motor signal functions, but beta oscillations are also prominent in somatosensory areas (Pfurtscheller and Lopes da Silva, 1999; Crone et al., 1998; Cheyne et al., 2003; Brovelli et al., 2004; Van Ede et al., 2010, 2011; 2012; Witham et al., 2007; Lebar et al., 2017). Our results (Torrecillos et al., 2015) do not tell whether the foreperiod beta modulation relates to efferent signals shaping the motor output or to mechanisms involved in the processing and integration of sensory information, critical for the adaptive update of the forthcoming movement (Krebs et al., 1998; Pavlides et al., 1993; Vidoni et al., 2010; Ostry and Gribble, 2016; Mathis et al., 2017).

In order to address these two connected issues, we used two complementary approaches. First, we exploited temporal independent component analysis (ICA) (Makeig et al., 1997; Delorme et al., 2012) to investigate the sources of the pre- and most-movement beta modulations. We used ICA to separate EEG activity into independent components (ICs) on which we performed time-frequency analyses. The patterns of beta-power modulations of relevant ICs, as well as the results of source analyses strongly suggest that distinct neural substrates underlie the modulations of the foreperiod and post-movement beta activities in response to kinematic errors.

Second, as a means of disentangling low-level efferent processes from higher-level information-integration processes, we contrasted bimanual reaching tasks involving physically similar movements but different action goals. The neural control of movement has been extensively studied using unimanual tasks or bimanual tasks involving bilateral separate, symmetrical or reciprocal, movements (Kelso et al., 1979; Marteniuk et al., 1984; Fowler et al., 1991; Donchin et al., 1998; Swinnen, 2002;
McCombe Waller and Whitall, 2008; Liuzzi et al., 2011). In contrast, cooperative tasks in which both hands have to achieve a common goal together have been rarely studied (for review, see Obhi, 2004). In a recent EEG study, Rueda-Delgado et al. (2017) examined changes in beta activity with task difficulty during a bimanual visuomotor task in which participants had to use both hands to draw a line, by rotating two dials (one with each hand) simultaneously. The difficulty of the task was manipulated through the ratio between the dial-rotation speeds. Against their expectations based on previous studies restricted to non-cooperative movements, Rueda-Delgado et al. (2017) found that only sensorimotor regions in the non-dominant right-hemisphere showed a modulation of beta power as a function of task difficulty. These results indicate that extending observations to cooperative movements offer an avenue to dissociate beta activity related to low-level efferent signals from beta activity related to higher-level information-integration processes.

Here, we contrasted beta oscillatory responses to movement errors in two bimanual reaching tasks involving physically similar movements, but different task goals. In a Parallel task, participants had to control two independent cursors (each with one hand) to reach simultaneously two different targets. In a Cooperative task, they controlled a single cursor with both hands to hit a unique target. In both cases, identical unilateral mechanical perturbations (force-field) were unexpectedly applied to one of the arms in a minority of trials (catch-trials), which elicited different patterns of trial-to-trial motor-command update, depending on the nature of the interlimb coordination. Consistent with the idea that it reflects adaptive processes, we found that the foreperiod beta activity was differently modulated in the two bimanual tasks. In contrast, the postmovement beta rebound exhibited no sensitivity to the nature of the interlimb coordination. More critically, we show that the foreperiod beta modulation does not relate to efferent signals shaping the motor output, but instead relates to upstream mechanisms likely involved in the integration of sensory information, essential for the adaptive updating of the upcoming movement.

\section{Material and methods}

\subsection{Participants}

A total of 15 healthy volunteers ( 9 males and 6 females, mean age 24.8 years, $\mathrm{SD}=3.9$ ) took part in the study. All participants were righthanded, as assessed by the Edinburgh Handedness Inventory (Oldfield, 1971) and all had normal or corrected-to-normal vision. All of them were free of neurological or psychiatric disorders and gave informed consent according to a protocol approved by the Ethics Board of the Aix-Marseille University.

\subsection{Experimental setup}

All tasks were performed using a robotic exoskeleton (KINARM, BKIN Technologies) that allows upper-limb movements in the horizontal plane and permits the application of mechanical loads to the elbow and shoulder joints (Fig. 1A). Participants were seated with both arms installed in the bilateral exoskeleton; the height of the chair was adjusted so that the shoulders were abducted by $\sim 70^{\circ}$. Using a semi-silvered mirror, direct vision of the arms was prevented throughout the task. One or two cursors (depending on the task) projected onto the same plane as the (not visible) hands provided movement visual feedback. Participants hold their hands in a fist, and the position of the cursor was determined by the position of the index knuckle. Head movements were limited by using a chin rest.

\subsection{Tasks}

Participants had to perform reaching movements to one or two visual targets depending on the task. In the initial configuration, the elbows 
A

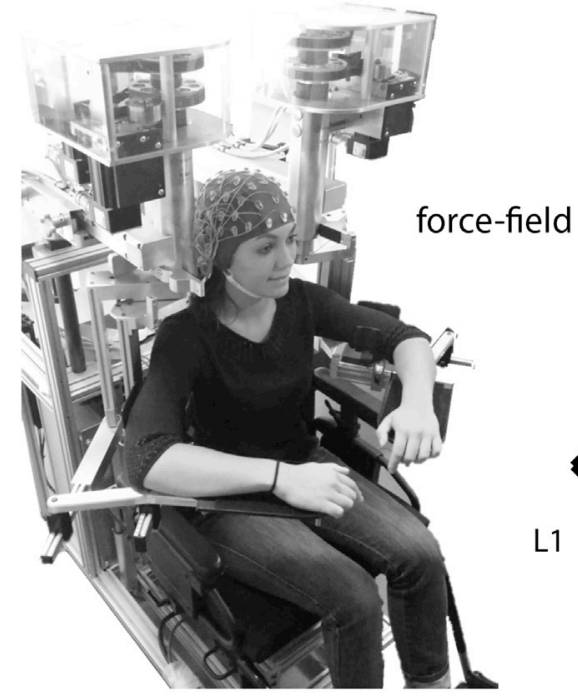

B

L1

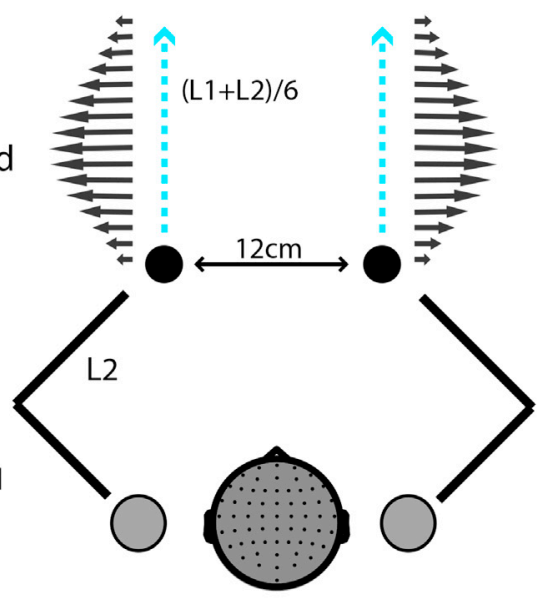

C

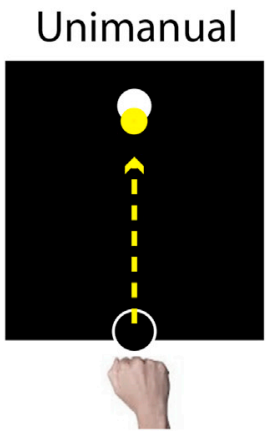

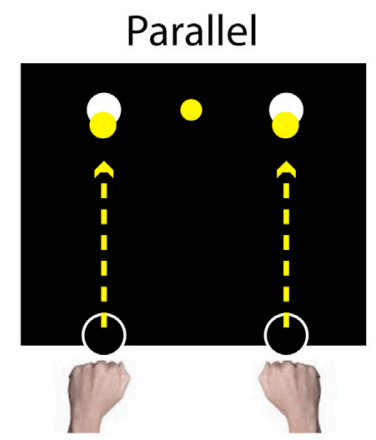

Parallel

\section{Cooperative}

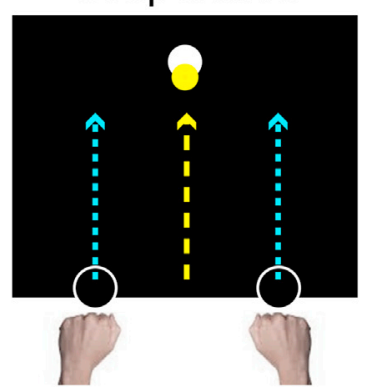

Fig. 1. Experimental setup.

A) EEG was recorded from participants performing reaching movement toward visual targets while installed in a bilateral exoskeleton that allows creating artificial force fields. B) In $20 \%$ of the trials (catch trials), a force-field was applied to one of the arm, in which the force was proportional and acted perpendicularly to the velocity of the hand. Half of the perturbations were applied to the left arm $(10 \%$ of the trials) and the other half to the right arm (10\% of the trials). The arrows indicate the directions of the forces that were applied to each arm. In the left-arm perturbation catch trials, the force acted counterclockwise, whereas as in the right-arm perturbation catch trials, they acted clockwise. C) Each participant performed three different reaching tasks. In a Unimanual task, they controlled a cursor (yellow dot) with their right (not visible) hand to reach a unique target (white dot). In a Parallel task, participants controlled two cursors, one with each hand, to reached two different targets. In a Cooperative task, they controlled with both hands a unique cursor to reach a unique target, whose position was defined as the average of the positions of the two hands (not visible hand trajectories are represented in cyan). In all tasks, participants were required to keep their eyes fixed. In the Unimanual and Cooperative tasks, they had to fixate the target, whereas in the Parallel task, a fixation point was displayed (yellow dot). The amplitudes of the movements were individually set, according to the length of the upper-arm (L1) and of the forearm (L2). were flexed at $\theta \mathrm{e}=90^{\circ}$, and the angles at the shoulders were about $\theta s=45^{\circ}$, varying slightly across participants, as they were individually adjusted so that the two start positions of the hands were separated by $12 \mathrm{~cm}$ (each hand $6 \mathrm{~cm}$ from the body midline). The distance between the starting position and the target(s) were also defined individually, calculated as $(\mathrm{L} 1+\mathrm{L} 2) / 6$, with $\mathrm{L} 1$ and $\mathrm{L} 2$ the upper-arm and the forearm length, respectively. The average reach amplitude was about $10 \mathrm{~cm}$ (Fig. 1B). The start locations and targets were indicated by white $1.5 \mathrm{~cm}$ diameter circles. Movement visual feedback was provided throughout the tasks.

The different reaching tasks are presented in Fig. 1C. In a Unimanual task, participants were instructed to reach a target with a cursor (yellow $1.0 \mathrm{~cm}$ diameter) controlled by their dominant-right hand. In a bimanual Parallel task, participants controlled two cursors (one with each hand) and had to reach two different targets simultaneously, whereas in a bimanual Cooperative task, they had to control a single cursor with both hands to hit a unique target. In this case, the cursor was presented at the spatial average position of the two hands.

The trials unfolded as follows. To initiate a trial, participants had to maintain their hand(s) in the start-circle(s) for $2000 \mathrm{~ms}$ before the target(s) appeared as (a) white circle(s) (READY). Following a $1500 \mathrm{~ms}$ delay, during which the hand(s) had to be kept still, the target(s) turned into (a) white disc(s) (GO). Participants were instructed to make reaching movements so as to reach the target(s) within of $375 \pm 25$ ms. Movement onset was defined as the time when the tangential velocity exceeded $7 \mathrm{~cm} / \mathrm{s}$. Movement-duration feedback(s) was/were provided at the time when the cursor(s) reached the target(s). The target(s) turned green, red or blue to indicate whether the movement(s) was/were too slow, too fast or had correct duration(s), respectively. In perturbation catch-trials (see below), no feedback about movement duration was provided, and the target(s) turned blue regardless of movement duration. Instructions about movement duration were provided in order to limit the variability in the amplitude of the perturbing force, defined as a function of movement speed. Importantly, the participants were clearly informed that they were not performing a reaction-time task and that they should take all the time they needed to plan movements executed within the prescribed time-window. After a delay of $1000 \mathrm{~ms}$, the arm(s) of the participants was/were passively brought back to its/their initial configuration(s). No visual hand-feedback was provided during the passive return(s) that lasted $1500 \mathrm{~ms}$. The cursor(s) and the start-circle(s) reappeared only when the hand(s) was/were back to its/their initial location(s). Each trial lasted about 7.0sec throughout which the participants were required not to move their eyes; in the Cooperative and the Unimanual tasks, they were required to keep fixating the target and in the Parallel task they had to keep fixating a fixation point $(0.4 \mathrm{~cm}$ diameter) projected between the two targets.

\subsection{Experimental protocol}

Movement-execution errors were induced by mechanical perturbations. As in previous studies (Torrecillos et al., 2014, 2015) the robotic device was programmed so as to create a curl-field in which the force is proportional and acts perpendicular to the velocity of the hand (Fig. 1B). The force was set to $\mathbf{f}=\mathbf{B} * \mathbf{v}$, where $\mathbf{f}=[\mathrm{fx}, \mathrm{fy}]$ depends on the velocity of the hand $\mathbf{v}=[v x, v y]$. For the perturbations applied to the left arm, $\mathbf{B}=[0,-\alpha, \alpha, 0] \mathrm{N}^{*} \mathrm{sec}^{* \mathrm{~m}}-1$, with $\alpha$ the force-amplitude parameter, so as to create a force-field acting counterclockwise. For the right $\operatorname{arm}, \mathbf{B}=[0$, $\alpha,-\alpha, 0] \mathrm{N} * \mathrm{sec}^{*} \mathrm{~m}-1$ so as to create a clockwise force-field. The field was produced by the torque-motors of the robotic exoskeleton, using the transformation from endpoint force to joint torque: $t=J^{\prime} * f$, where $\mathrm{J}=[\mathrm{L} 1 * \sin (\theta \mathrm{s})+\mathrm{L} 2 * \sin (\theta \mathrm{s}+\theta \mathrm{e}), \quad \mathrm{L} 2 * \sin (\theta \mathrm{s}+\theta \mathrm{e}) ; \quad \mathrm{L} 1 * \cos (\theta \mathrm{s})+$ $\mathrm{L} 2 * \cos (\theta \mathrm{s}+\theta \mathrm{e}), \mathrm{L} 2 * \cos (\theta \mathrm{s}+\theta \mathrm{e})]$ is the arm-configuration-dependent 
differential transformation matrix (Jacobian matrix).

The experiment consisted of two experimental sessions, run on two different days separated by at least 2 days. In each experimental session, after the calibration of the robotic device and verbal instructions about the task requirements, participants first performed one of the two bimanual tasks (Parallel or Cooperative) and then performed the Unimanual task. The order of the sessions was counterbalanced across the participants. Each session included, for each task, a preliminary familiarization phase, during which the participants learned the task, and an experimental phase, during which they performed the task and EEG activity was recorded.

Preliminary phases. The participants learned each task performing 1 block of 50 reaching movements including $20 \%$ perturbation catch-trials in which reach errors were induced by mechanical perturbations applied to one of the two arms. Depending on the sizes of the hand-path deviations that were observed in the catch-trials interspersed in this familiarization block, the force-amplitude parameter was set to $\alpha=7$ or 8 , for 6 and 9 participants respectively. The same force-amplitude was then used for all the tasks of the two sessions.

Experimental phases. The bimanual (Parallel and Cooperative) tasks consisted of 6 blocks of 100 trials each, including 80 trials interspersed with $20 \%$ catch-trials. Half of the perturbations were applied to the left arm (10\% of the trials) and the other half to the right arm (10\% of the trials). The Unimanual task counted 3 blocks of 100 trials, in which all perturbations ( $20 \%$ of the trials) were applied to the moving right arm. In all cases, two catch-trials were always separated by at least three noperturbation trials.

Each block lasted about $12 \mathrm{~min}$, and breaks of about $2 \mathrm{~min}$ were allocated between blocks. One break of about 30min separated the two tasks (Bimanual and Unimanual tasks) for an experimental session lasting about $4 \mathrm{~h}$ in total (including the calibration of the robot and EEG installation).

Note that we were aware that whilst, in the unperturbed trials, kinematics was similar across the two bimanual tasks, differences in the visual information remained inherent to the tasks: in Parallel task, two cursors were displayed, whereas in the Cooperative task visual feedback was provided by a single cursor. However, Diedrichsen (2007) has demonstrated that the behavioral task effects were not due to these differences. In his experiments, the visual feedback of the cursor was withdrawn in half of the trials, making the one- and two cursor conditions identical except as for task instructions. Even without visual feedback, participants showed similar task-related behavioral differences. This is why to limit the duration of the experimental sessions we decided not to include any additional control trials.

\subsection{Behavioral data recording and analysis}

For both arms, the angular position and velocity data of the motor resolvers were collected at $1000 \mathrm{~Hz}$. These signals were down-sampled to $100 \mathrm{~Hz}$ offline, and then filtered with a 2nd order zero-phase-shift lowpass Butterworth filter (cut-off frequency of $10 \mathrm{~Hz}$ ). Hand position and velocity were calculated from these angular data. Kinematic data were analyzed using custom routines written in Matlab R2015b (The MathWorks). The trials in which the hand(s) was/were not maintained stable enough (tangential velocity $>6 \mathrm{~cm} / \mathrm{s}$ ) in the start-position during the delay between the READY and GO signals, or in which the movement(s) was/were initiated before the GO signal were excluded from the analyses ( $\sim 5 \%$ of trials). Movement end was defined offline as the time when the tangential velocity fell below $5 \mathrm{~cm} / \mathrm{s}$.

In view of analyzing the error-related beta responses observed during the pre- and the post-movement periods, for all three tasks (Parallel, Cooperative, and Unimanual) we divided the recorded data into three subsets. The first subset was used to assess the impact of reach error on the post-movement beta rebound and included the catch-trials $n$. The second data subset was used to assess the error-related modulation of the foreperiod beta activity, and included the reaches $n+1$ (after-trials) that followed directly a deviated movement $n$. A third subset consisted of the unperturbed trials $n-1$, which served as baseline to which the trials $n$ and $n+1$ would be compared. These latter trials were used as baseline since they offered the warranty of not having been influenced by a perturbation applied in the preceding trial.

We used two different measures to quantify, respectively, the handpath deviations induced in the perturbation catch trials $n$ and the amplitudes of the motor-command updates visible as slight deviations in the opposite direction (after-effects), in the following trials $n+1$. For trials $n$, we measured the maximum perpendicular hand-path deviation (PD-max) to quantify the hand-path "hooks" characterizing these movements. For the after-trials $n+1$, we used as kinematic index the hand-path deviation at the peak of the tangential velocity (PD-vel) to capture the error in the initial movement direction (feedforward component), as it reflects the adaptive update of the motor-command. For each task, both deviation measures were corrected for individual reach-direction biases by subtracting from each trial measure the mean of the deviations observed in the baseline trials $(n-1)$.

Univariate statistical tests were run, on the different kinematic measures, using Matlab R2015b (The MathWorks). Paired t-tests were used for the Unimanual task (in which only the right arm is perturbed), and repeated-measures two-way ANOVAs were run for the bimanual (Parallel and Cooperative) tasks (in which the left or the right arm is perturbed). For all tests, the significance threshold was set to 0.05 and corrected with the Bonferroni procedure for complementary pairwise comparisons.

\subsection{EEG data recording and analysis}

EEG activity was recorded continuously at $1024 \mathrm{~Hz}$ using a 64-channel Biosemi ActiveTwo system (BioSemi) referenced to the Common Mode Sense-Drive Right Leg (CMS-DRL) ground. Electrodes were embedded into an elastic cap and distributed over the scalp according to the extended 10-20 EEG system. For each participant, electrode locations and nasion and preauricular points were recorded by an infrared camera (Rogue Research). Electro-oculographic (EOG) activity was recorded with surface electrodes placed near both outer canthi (saccades) as well as under and above the right orbit (blinks).

EEG preprocessing. EEG data preprocessing was performed using the free software ELAN (Aguera et al., 2011), which allows especially good visual inspection of the raw EEG signals for artefact rejection purposes. Continuous signals were re-referenced to the average of all electrodes, filtered between 0.5 and $100 \mathrm{~Hz}$ (Butterworth order 2) and down-sampled to $256 \mathrm{~Hz}$. Non-stereotypical artifacts (that cannot be captured by ICA) were identified and rejected upon visual data screening. Further analyzes were run using the free and open-source softwares Fieldtrip (Oostenveld et al., 2011) and EEGlab (Delorme and Makeig, 2004).

Independent Component Analysis (ICA). The preprocessed EEG signals were cut into time-segments extending from -4000 to $+3500 \mathrm{~ms}$ with respect to reaching-movement onset of the right-dominant arm, which covered the complete trials, slightly variable in duration. As the movement onsets of the left and right arms were separated by only $7 \pm 1 \mathrm{~ms}$ in the Parallel task and $6 \pm 2 \mathrm{~ms}$ in the Cooperative task (Parallel task, for 9 participants the right arm preceding the left arm; Cooperative task, for 8 participants the right arm preceding the left arm), and the movement offsets of the left and right arms were separated by only $12 \pm 5 \mathrm{~ms}$ in the Parallel task and $31 \pm 10 \mathrm{~ms}$ in the Cooperative task (Parallel task, for 12 participants the left arm preceding the right arm; Cooperative task, for all participants the left arm preceding the right arm) we arbitrarily present here the results for the signals aligned relative to the kinematics of the right-dominant arm. The epoched EEG data of the different tasks were then submitted to separate ICA (runica algorithm) as they were acquired on separate days. For the Unimanual task, the data acquired during the first experimental session were analyzed.

EEG time-frequency analysis. Time-frequency analyzes were performed on the time-courses of the independent components (ICs). Single-trial 
signals were transformed in the time-frequency domain by convolution with the complex Morlet's wavelets characterized by the ratio $\mathrm{fO} / \sigma \mathrm{f}=7$, with f0 ranging from 10 to $35 \mathrm{~Hz}$ by steps of 0.5 .Hz. For each task, in order to calculate the event-related changes in beta power, the raw power data was log-transformed and then normalized relative to the average power calculated over all trials, as no clear baseline could be defined during the task (Tan et al., 2014; Torrecillos et al., 2015). For each type of trials (trials $n$, after-trials $n+1$ and no-perturbation trials $n$ - 1 ) and each individual, for each time point ( $50 \mathrm{~ms}$ bin), power was averaged over trials within a specific beta frequency band (see below) and smoothed using a Gaussian Kernel with 7 -time points $(350 \mathrm{~ms})$ full-width at half maximum. For each task and for each participant, the same number of perturbation catch trials $n$, after-trials $n+1$ and no-perturbation trials $n-1$ were used for the statistical analyses. For the Unimanual task, on average $51 \pm 5$ trials were included for each participant. For the two bimanual tasks, the perturbation catch trials $n$ and the after-trials $n+1$ were divided according to the arm to which the perturbation was applied: left-arm perturbed $(L P)$ or right-arm perturbed $(R P)$. On average, for each participant, $48 \pm 5$ and $48 \pm 6$ trials were used for each perturbation condition (left-arm perturbed $(L P)$ or right-arm perturbed (RP)) in the Parallel and Cooperative tasks, respectively. For these trials, the repartition between the trials that were "too fast", "too slow" or of "correct" duration was as follows: for the Unimanual task, $10 \pm 4,20 \pm 3,21 \pm 4$, respectively; for the Cooperative task, $5 \pm 2,19 \pm 4,24 \pm 4$, respectively; for the Parallel task, $5 \pm 3,17 \pm 5,26 \pm 4$, for the right arm and $7 \pm 4$, $16 \pm 5,24 \pm 4$, for the left arm.

Independent Components (ICs) selection. Our goal was to identify, for each participant, ICs that best accounted for the attenuation of the postmovement beta rebound (in perturbation catch-trials $n$ ) or for the modulation of the foreperiod beta power profiles (in after-trials $n+1$ ). For this purpose, we examined the (time-invariant) topographies and timefrequency representations of the time courses of the ICs, and considered our previous observations (Torrecillos et al., 2015) in a unimanual reaching task, identical to the one used here (but for the position of the hand, pointing instead of making a fist). In this previous study, first, the beta rebound was observed more medially than the transient foreperiod beta-power enhancement, clearly contralateral to the moving arm. Second, the beta rebound was observed around $500 \mathrm{~ms}$ after the end of the movement, and the foreperiod beta enhancement peaked around $1000 \mathrm{~ms}$ before reach onset (see Torrecillos et al., 2015, Fig. 9B). Based on these features, we defined spatial regions of interest (ROIs) and time-windows of interest. First, we defined a large medial ROI including 15 electrodes (F1-Fz-F2-FC1-FCz-FC2-C1-Cz-C2-CP1-CPz-CP2-P1-Pz-P2) and two lateral ROIs (one for each hemisphere) including each 9 electrodes (left hemisphere: C5-C3-C1-CP5-CP3-CP1-P5-P3-P1; right hemisphere: C2-C4-C6-CP2-CP4-CP6-P2-P4-P6). Second, we defined two distinct time-windows of interest, delimited relative to movement offset and movement onset respectively: a post-movement-window (1000 ms wide) centered at $500 \mathrm{~ms}$ after movement offset, and a foreperiod-window (700 ms wide) centered at $1000 \mathrm{~ms}$ before movement onset. The widths of the two time-windows of interest were defined in relation to the observed durations of the two beta power enhancements.

For each participant, we selected for each task, one "medial IC", as well as two lateral ICs (one for each hemisphere, "left IC" and "right IC") proceeding in two steps (pre-selection and final selection). To select the medial IC, first we pre-selected one to three (depending on the participant) IC(s) upon examination of its/their topographies; the IC(s) exhibiting largest weighting within the 15-electrodes medial ROI was/were pre-selected. In a second step, we examined the time-frequency representation of the time-course of the pre-selected IC(s) observed in the noperturbation (NP) trials $n-1$. Based on the time-frequency maps and the corresponding beta power profiles (obtained by averaging over $4 \mathrm{~Hz}-$ wide frequency bands centered about frequencies 13 to 33 by steps of $2 \mathrm{~Hz}$ ), we selected the IC and the frequency-band exhibiting the largest beta power increase during the post-movement-window. To select the left and right lateral ICs, first we pre-selected for each hemisphere the
IC(s) that exhibited largest weighting within the 9-electrodes lateral ROIs, one or two (depending on the participant). Then, based on the time-frequency maps and the corresponding beta power profiles (also for the no-perturbation (NP) trials $n$-1) we selected, for each hemisphere, the IC and frequency-band exhibiting the largest local beta power peak within the foreperiod-window. Thus, in each case, the frequency bands were individually selected. Fig. 2 shows the ICs selected for one participant.

Statistical analyzes of the beta power profiles of the ICs. Statistical analyzes were performed using Matlab R2015b (The MathWorks). We proceeded to two main ensembles of comparisons. First, we contrasted the beta power profiles of the ICs observed for the different perturbation conditions (left-arm perturbed $(L P)$, right-arm perturbed $(R P)$ and noperturbation $(N P)$ ) for each reaching task separately. In a second ensemble of comparisons, we contrasted the no-perturbation (NP) trials $n-1$ used as baseline across the different tasks. In all cases, the beta power profiles of the ICs were analyzed within the same post-movement and foreperiod time-windows of interest that were used to select the ICs. For the comparisons within the post-movement window, we considered the perturbation catch trials $n$ and the baseline trials $n-1$, whereas for the contrasts within the foreperiod window, we considered the trials $n+1$ and the baseline trials $n-1$. In all cases, at each time point ( $50 \mathrm{~ms}$ steps) of the time-window of interest, we computed a paired $t$-test or a repeatedmeasures ANOVA. The False Discovery Rate (FDR) method was used to correct for multiple comparisons along the time axis. ANOVAs were followed, at each significant time-point, by pairwise comparisons corrected for multiple comparisons by the Bonferroni procedure.

In addition, we compared the frequency bands individually selected for the foreperiod and the beta rebound, running a two-way repeatedmeasures ANOVA with the task (Unimanual, Parallel and Cooperative) and the time-period (pre- or post-movement) as within-subject factors. In all cases, the significance level was set to 0.05 .

Dipole fitting and Measure Projection Analysis (MPA) of the ICs. For each participant, an equivalent current dipole model was computed for each selected IC using DIPFIT EEGLAB plug-in. Using the recorded locations of the EEG electrodes, dipoles were localized within a three-shell boundary element model (BEM) of the Montreal Neurological Institute (MNI) standard brain. For all participants, except one whose data were excluded, all selected ICs had one or two dipole solutions with residual variance smaller than $10 \%$. Measure projection analysis (MPA) was then applied using the EEGLAB plug-in (Bigdely-Shamlo et al., 2013). MPA is a parsimonious method for identification of brain regions (domains) exhibiting statistically significant EEG-measure homogeneity. These domains are identified in a data-driven manner with unique EEG-measure (e.g., ERSP) features in 3D space.

The standard deviation of the three-dimensional Gaussian representing each dipole location distribution was set to $8 \mathrm{~mm}$. Each Gaussian that represented a dipole was truncated to a radius of 3 standard deviations to prevent influences from distant dipoles. To define anatomical regions, the toolbox incorporates the probabilistic atlas of human cortical structures provided by the LONI project (Shattuck et al., 2008). To create the domains, a cubic space grid with 6-mm spacing was situated in the brain volume in MNI space which served as the MPA brain model. Local convergence (homogeneity) values were calculated based on the algorithm developed by Bigdely Shamlo et al. (2013), which deals with multiple comparisons problem. A pairwise IC similarity matrix is constructed by estimating the signed mutual information between event-related power-spectral perturbation (ERSP) measures of the individual ICs. Here, maximal exemplar-pair similarity (forcing creation of additional clusters) was set to a correlation value of 0.8 , and the outlier detection similarity threshold to a correlation value of 0.7 . A significance threshold for convergence at each brain location is obtained by bootstrap statistics. The raw voxel significance level was set to 0.05 .

IC dipole fitting and MPA were applied for each task, Unimanual, Parallel and Cooperative, separately, for which only the unperturbed trials (n-1) were considered. 
A
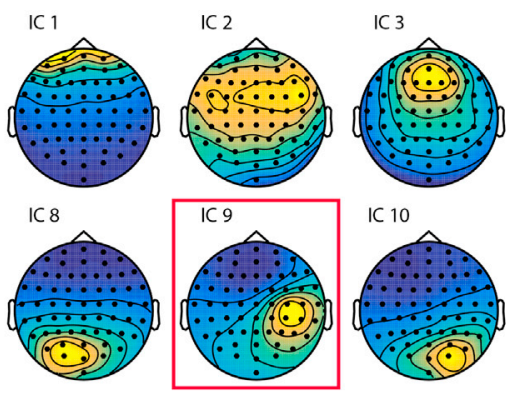

IC 10
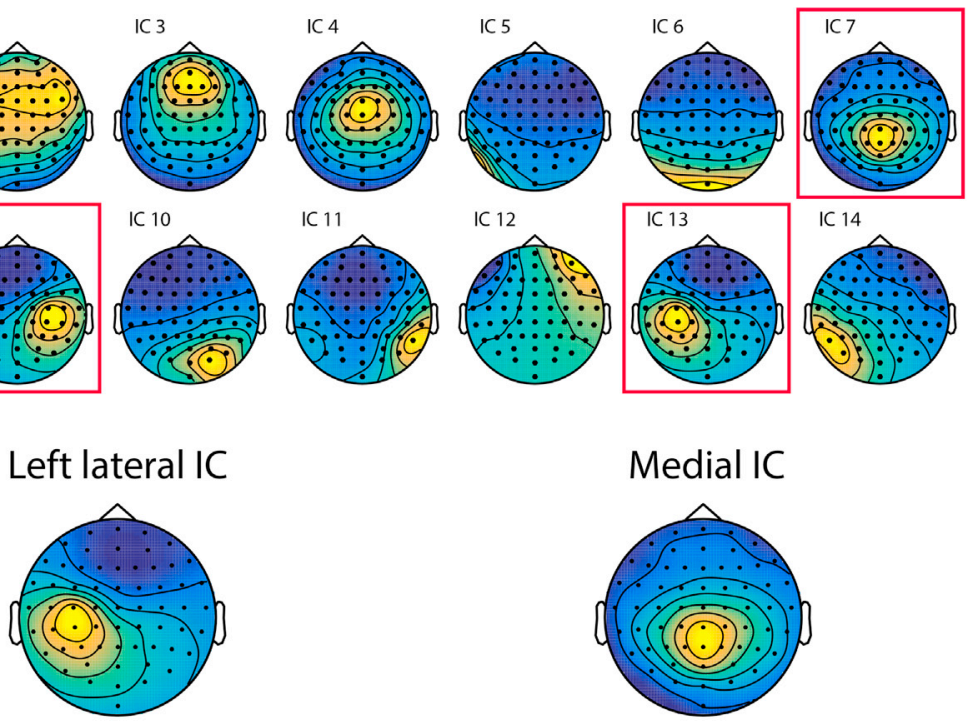

B
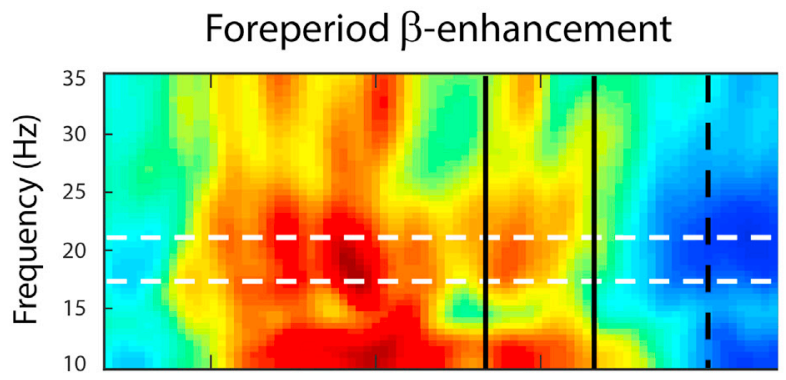

C

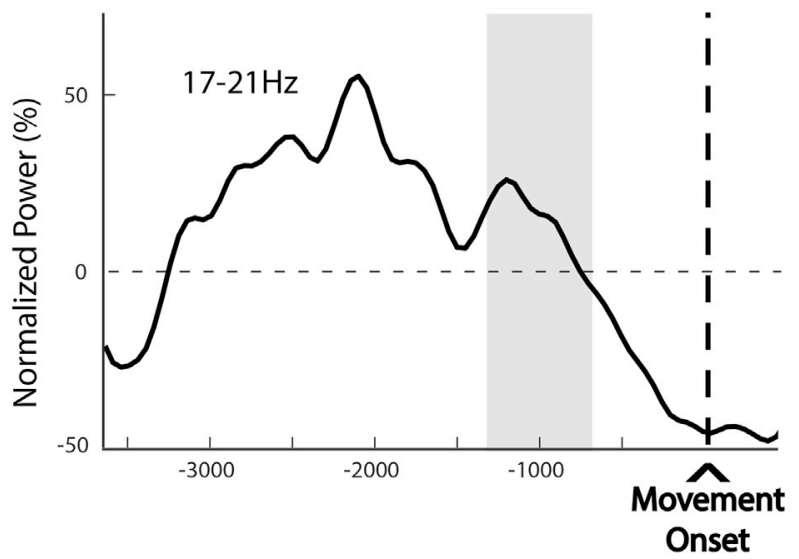

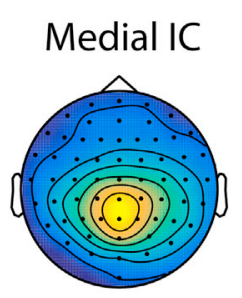

$\beta$-rebound
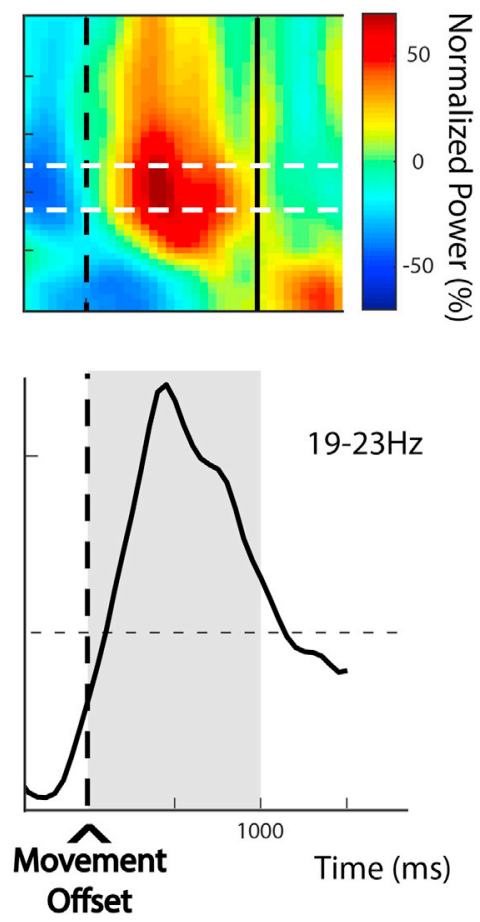

Fig. 2. Illustration of independent components (ICs) selection.

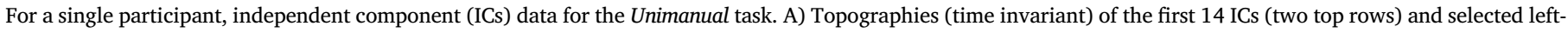

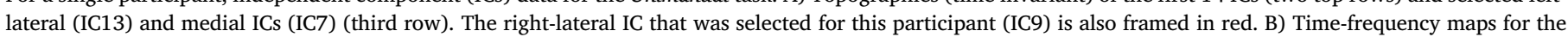

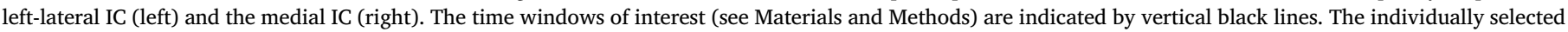

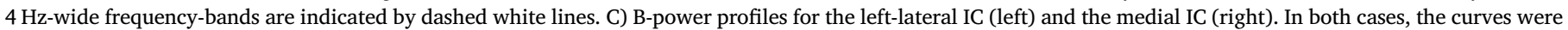
obtained by averaging over the individually selected $4 \mathrm{~Hz}$-wide frequency-bands. The gray shadings show the time-windows of interest (see Methods).

\section{Results}

\subsection{Behavioral performance}

Perturbation Catch Trials n. During the reaching tasks, a mechanical perturbation (force-field) was unexpectedly applied in $20 \%$ of the trials. It was applied on the right-dominant arm in the Unimanual task, and on the left $(10 \%)$ or the right $(10 \%)$ arm in the bimanual tasks. As expected, in all reaching tasks, clear hand-paths deviations were induced for the arm to which the perturbation was applied. However and consistent with previous findings (Diedrichsen, 2007), contrasting patterns were observed for the two bimanual tasks. In the Parallel task only the perturbed arm exhibited deviated hand-paths, whereas in the Cooperative task deviations were also observed for the opposite arm to which no mechanical perturbation was directly applied. This is illustrated by the individual hand-paths plotted in Fig. 3. Kinematic errors were quantified by the maximum perpendicular deviations (PD-max) reflecting the amplitudes of the "hooks" induced in the hand-paths. To be submitted to statistical tests, these measures were corrected for individual biases in movement directions, assessed in the baseline trials $n-1$ (see Methods). 
A Parallel task

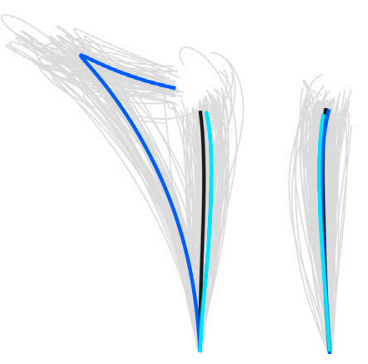

Left-arm perturbation

\section{B Cooperative task}

$$
\begin{aligned}
& \text { - baseline trials } n-1 \\
& \text { catch-trials } n \\
& \text { after-trials } n+1
\end{aligned}
$$
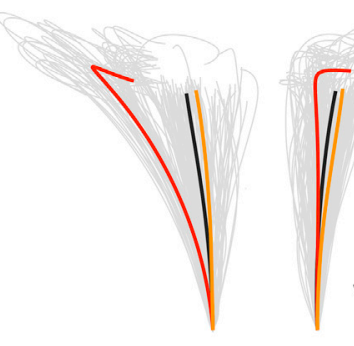

Left-arm perturbation
Right-arm perturbation

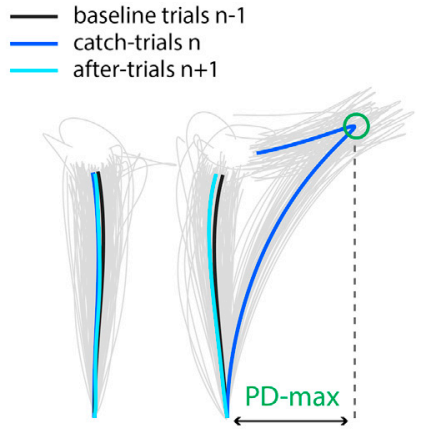

Fig. 3. Illustrative behavioral data for the Parallel and Cooperative tasks. For a single participant, the mean hand-paths of the different trial subsets (catch-trials $n$, after-trials $n+1$, and baseline trials $n$ - 1 ) are plotted in thick lines. Individual trials are represented in light gray. For group analyses, different indices were used to quantify kinematic-error in the catch-trials $n$ and after-trials $n+1$. The maximum perpendicular deviations (PD-max) reflecting the amplitudes of the hooks induced in the hand-paths was used for the catch trials $n$. Whereas the perpendicular deviation at peak velocity (PD-vel) that captures the initial (feedforward component) error, reflecting the adaptive updates of the motor-command, was used for the after-trials $n+1$. A) In the Parallel task, the unilateral perturbations (left-arm or right-arm perturbations) induced hand-path deviations in the catch-trials $n$ (in dark blue) only for the arm on which the force-field was applied. Similarly, adaptive motor-command updates (after-effects) visible in the subsequent trials $n+1$ (in light blue) as slight hand-path deviations in the opposite direction were manifest for the perturbed arm only. B) For the Cooperative task, in the catch-trials $n$ (in red-orange) deviations are also induced in the trajectory of the arm on which the force-field was not applied (Unperturbed arm). Similarly, in the subsequent trials $n+1$ (in yelloworange) after-effects were visible for both arms.

The results of the group analyses are summarized in Fig. 4. We proceeded to separate analyzes for the Unimanual and bimanual tasks. For the Unimanual task, we conducted a $t$-test which confirmed that reaches in trials $n$ were significantly deviated relative to the baseline movements $n-1\left(\mathrm{t}_{(14)}=-18.43, \mathrm{p}<0.0001\right)$. For the bimanual tasks, our aim was to test whether different hand-paths deviations were observed for the perturbed arm (directly submitted to the force-field) and the opposite unperturbed arm (not directly submitted to the force-field) depending on the nature of the interlimb coordination, Parallel or Cooperative. In order to proceed to this test, we considered together data of both arms, which we categorized as Perturbed or Unperturbed. Positive PD-max values were associated to deviations in the same direction as the applied force-field; that is, leftward deviations for the left arm and rightward deviations for the right arm.

In a preliminary step, we checked that movements performed in the absence of perturbation had similar kinematics in both bimanual tasks.

\section{A Catch-trials $n$}

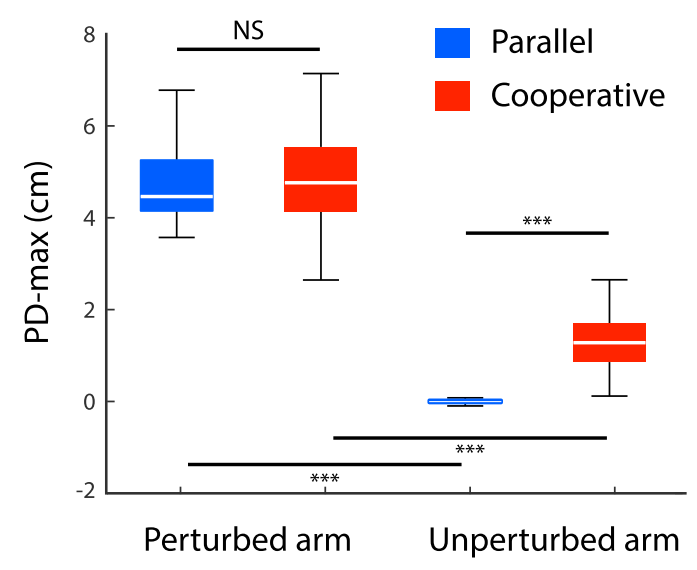

\section{B After-trials $\mathrm{n}+1$}

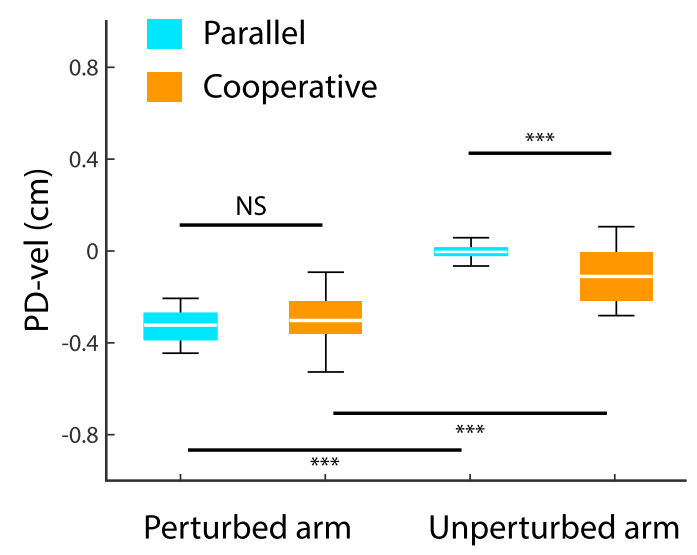

Fig. 4. Group behavioral data for the Parallel and Cooperative tasks. Boxplots summarize group data for the two bimanual tasks. Statistically significant differences are indicated by $* * * p<0.001$. A) The hand-paths hooks induced in the catch trials $n$ were quantified by the maximum perpendicular deviations (PD-max, see Fig. 3). B) The initial (feedforward component) error, reflecting the adaptive updates of the motor-command, in the after-trials $n+1$ were quantified by the perpendicular deviation at peak velocity (PD-vel, see Fig. 3).

For each arm, considering the baseline no-perturbation trials $n-1$ only, we tested the effect of the task (Parallel versus Cooperative) on the hand-pathdeviations. No significant difference between the two tasks was revealed

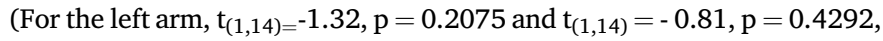
for PD-vel and PD respectively. For the right arm, $t_{(1,14)=}-0.68$, $\mathrm{p}==0.5096$ and $\mathrm{t}_{(1,14)}=1.03, \mathrm{p}=0.3218$, for PD-vel and PD-max respectively). We ran a repeated-measures two-way ANOVA on the bias-corrected PD-max, with Task (Parallel versus Cooperative) and Arm (Perturbed versus Unperturbed) as within-subject factors. The differential effect of the unilateral perturbations depending on the interlimb coordination was confirmed by a significant Task $\times$ Arm interaction $\left(F_{(1,14)}=12.77, p=0.0031\right)$. We completed this result by testing for simple effects. First, as expected, in both bimanual tasks significantly larger deviations were induced in the Perturbed than in the Unperturbed arm (Parallel task: $\mathrm{t}_{(14)}=20.09$ and Cooperative task: $\mathrm{t}_{(14)}=13.41$, $\mathrm{p}<0.05$ in both cases). Then, considering the data for the Perturbed arm only, the sizes of the induced deviations did not differ significantly between the tasks $\left(t_{(14)}=-0.31, p>0.05\right)$. In contrast, a significant simple effect of the task was revealed for the Unperturbed arm, with unilateral 
mechanical perturbations inducing significantly larger hand-path deviations in the Cooperative than in the Parallel task $\left(t_{(14)}=-6.03\right.$, $\mathrm{p}<0.05$ ).

After-Perturbation Trials $\mathrm{n}+1$. In addition to the clear reach errors observed in the perturbation catch-trial $n$, slight hand-path deviations in the opposite direction were visible in the subsequent trials $n+1$, consistent with previous findings (Thoroughman and Shadmehr, 2000; Torrecillos et al., 2015). Fig. 3 shows individual hand-paths for one participant. To quantify these slight deviations, we measured the perpendicular deviations at peak velocity (PD-vel) which captures the initial (feedforward component) errors in the reach trajectories, reflecting the adaptive updates of the motor-command. These measures were corrected for individual biases quantified in the baseline no-perturbation trials $n$-1. The amplitudes of the deviations were in accordance with our previous observations (Torrecillos et al., 2015). We submitted this data to a two-way repeated-measures ANOVA, which revealed a significant Task $\times$ Arm interaction effect $\left(\mathrm{F}_{(1,14)}=5.73, \mathrm{p}=0.0312\right)$. To complete this result we tested for simple effects. First in each bimanual task, significantly larger deviations (after-effects) were observed for the arm Perturbed in the previous trial than in the Unperturbed arm (Parallel task: $\mathrm{t}_{(14)}=-13.65$ and Cooperative task: $\mathrm{t}_{(14)}=-3.74, \mathrm{p}<0.05$ in both cases). Testing on the data of the Perturbed arm only revealed no significant simple effect of the task $\left(\mathrm{t}_{(14)}=-0.56, \mathrm{p}>0.05\right)$, although slightly larger deviations (after-effects) were observed for the Parallel than for the Cooperative task. In contrast, deviations of different amplitudes were observed in the two bimanual tasks for the Unperturbed arm, with significantly larger after-effects induced in the Cooperative than in the Parallel task $\left(\mathrm{t}_{(14)}=3.65, \mathrm{p}<0.05\right)$.

Also, it was important to check that changes in the transient betapower enhancement could not be due to increased variability in the delays between the GO signal and the movement onset in the trials $(n+1)$ following a perturbation, relative to the baseline trials $(n-1)$. (Note that we do not use the term "reaction-time" since the tasks were performed with no time pressure.) For the three tasks (Unimanual, Parallel and Cooperative) we assessed the equality of the delay variances between the two types of trials ( $n+1$ versus $n-1)$; Levene's tests for equality of variances did not reveal any significant difference (Unimanual: $\mathrm{F}(1,1702)=0.5377, \quad \mathrm{p}=0.4635 ; \quad$ Parallel: $\mathrm{F}(1,3428)=0.0222$, $\mathrm{p}=0.8817$; Cooperative: $\mathrm{F}(1,3450)=0.8884, \mathrm{p}=0.3460)$.

In summary, as expected, in all three reaching tasks clear hand-paths deviations were observed in the perturbation catch trials $n$ for the arm to which the perturbation was applied. Also consistent with our previous observations (Torrecillos et al., 2015), in all cases the post-perturbation movements $n+1$ by the arm that was perturbed in the previous trial exhibited slight deviations in the direction opposite to the force-field (after-effects). More interestingly and consistent with Diedrichsen's findings (2007) the unilateral mechanical perturbations elicited different adaptive responses in the two bimanual tasks. In the Parallel task deviated hand-paths were visible for the arm submitted to the force-field only, whereas in the Cooperative task reach deviations were also observed for the opposite arm, on which no mechanical perturbation was applied.

\subsection{EEG time-frequency data}

In Figs. 5-8, data are shown for the different tasks separately. The group averaged topographies for the different selected independent components (ICs), "medial IC", "left IC" and "right IC", are presented along with the corresponding beta power profiles. For each perturbation condition ( $L P, R P$ and $N P$ ), the group average beta power profiles were obtained from individual power-profiles computed over $4 \mathrm{~Hz}$-wide individually selected frequency-bands (see Materials and Methods). The frequency bands individually selected did not differ significantly between the pre- and the post-movement period $\left(\mathrm{F}_{(1,14)}=1.9, \mathrm{p}=0.19\right)$, or across the tasks $\left(\mathrm{F}_{(2,14)}=0.16, \mathrm{p}=0.85\right)$, and no significant interaction effect was observed $\left(\mathrm{F}_{(2,14)}=1.71, \mathrm{p}=0.20\right)$ (For the Unimanual task, foreperiod: $20.47 \pm 1.16 \mathrm{~Hz}$, post-movement: $20.6 \pm 1.19 \mathrm{~Hz}$; for the Parallel task: foreperiod: $21.96 \pm 1.19 \mathrm{~Hz}$, post-movement: $20.33 \pm 1.18 \mathrm{~Hz}$; for the Cooperative task: foreperiod: $22.33 \pm 1.17 \mathrm{~Hz}$, post-movement: $19.4 \pm 1.37 \mathrm{~Hz}$ ).

Post-movement beta rebound. For all reaching tasks, the group average beta power profiles of all ICs exhibited a clear post-movement beta rebound around $500 \mathrm{~ms}$ in all conditions. First, for each task separately, we tested whether post-movement beta power was modulated by the movement-execution errors experienced in trials $n$. At each time point within the post-movement-window of interest (see Materials and Methods; indicated by a gray shading in Figs. 5-6), we computed a $t$-test (for the Unimanual task, Fig. 5) or repeated-measures ANOVA (for the bimanual tasks, Fig. 6) with the perturbation condition, left- or right-arm perturbation ( $L P$ or $R P$ ) and no-perturbation $(N P)$, as within-subject

\section{Post-movement $\beta$-rebound}
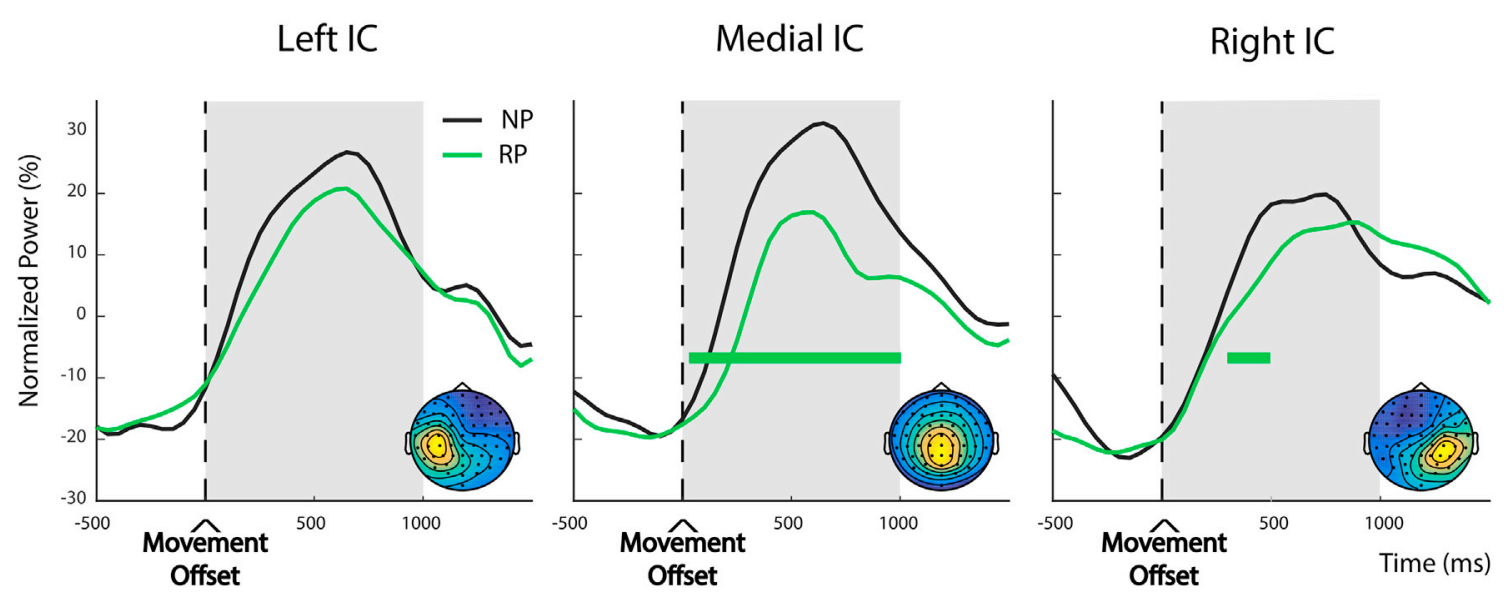

Fig. 5. Modulations of the post-movement beta rebound in the catch-trials $\boldsymbol{n}$ for the Unimanual task.

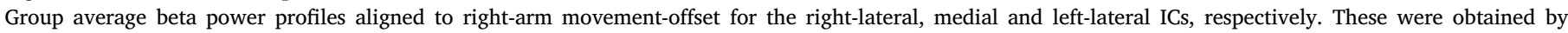

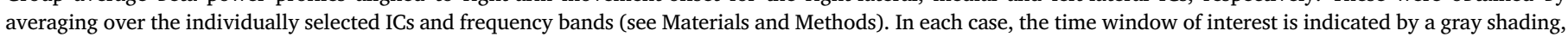

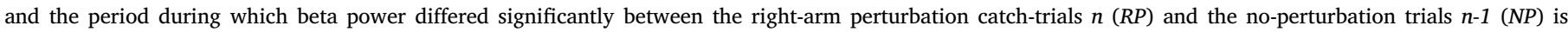
indicated by colored bars. In each plot, the group average (time invariant) topographies of the selected ICs are presented. 


\section{Post-movement $\beta$-rebound}

\section{A Parallel}

Left IC

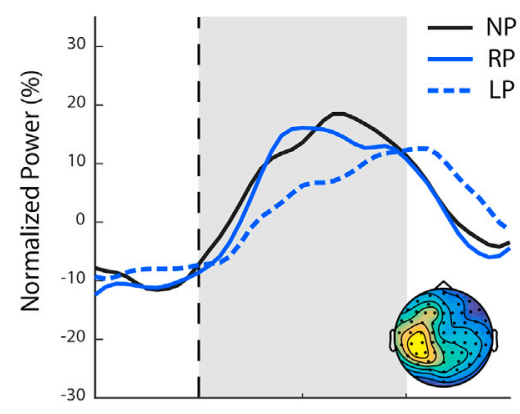

Medial IC

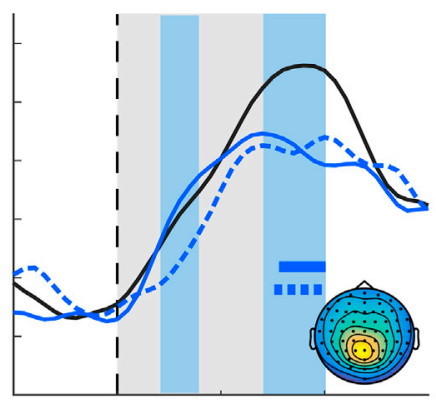

Right IC

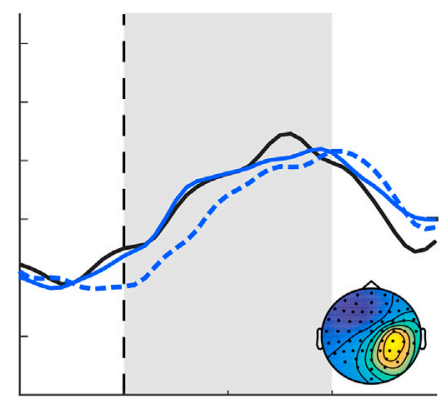

\section{B Cooperative}
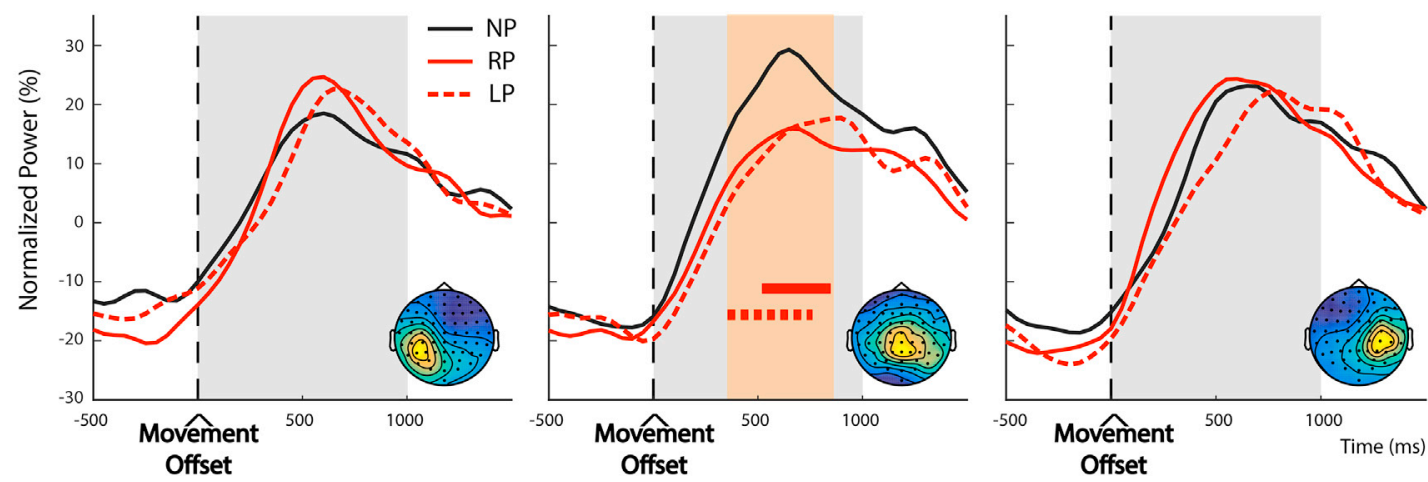

Fig. 6. Modulations of the post-movement beta rebound in the catch-trials $\boldsymbol{n}$ for the Parallel and Cooperative tasks.

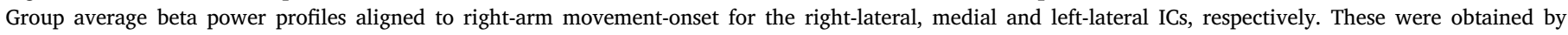

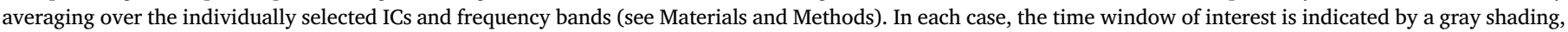

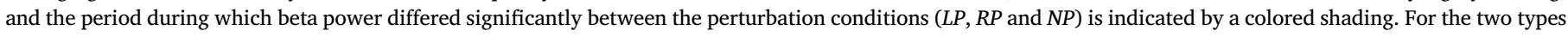

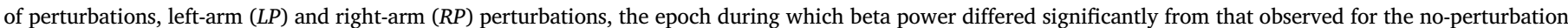

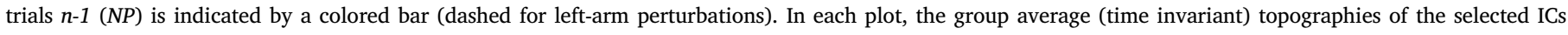
are presented.

\section{Foreperiod $\beta$-enhancement}
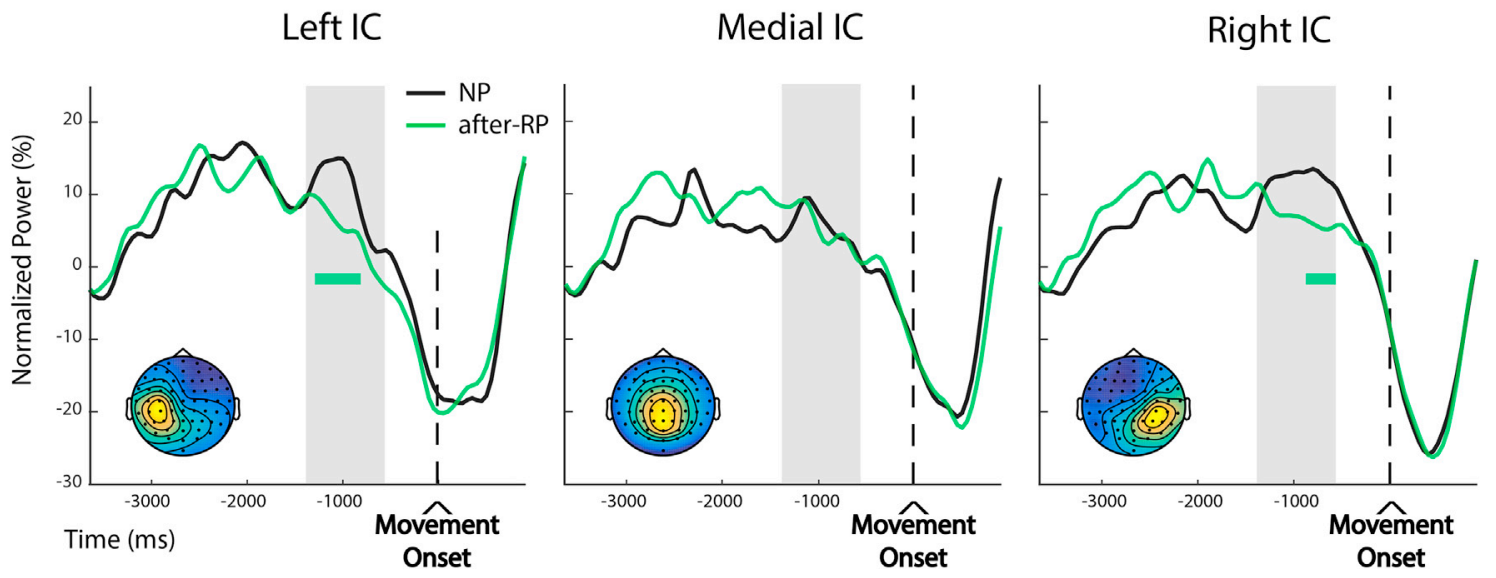

Fig. 7. Modulations of the foreperiod beta activity in the after-trials $n+1$ for the Unimanual task.

Group average beta power profiles aligned to right-arm movement-onset for the right-lateral, medial and left-lateral ICs, respectively. These were obtained by averaging over the individually selected ICs and frequency bands (see Materials and Methods). In each case, the time window of interest is indicated by a gray shading, and the period during which beta power differed significantly between the after-trials $n+1$ that followed a right-arm perturbation (after-RP) and the no-perturbation trials $n-1(N P)$ is indicated by colored bars. In each plot, the group average (time invariant) topographies of the selected ICs (the same as in Fig. 5) are presented. 


\section{Foreperiod $\beta$-enhancement}

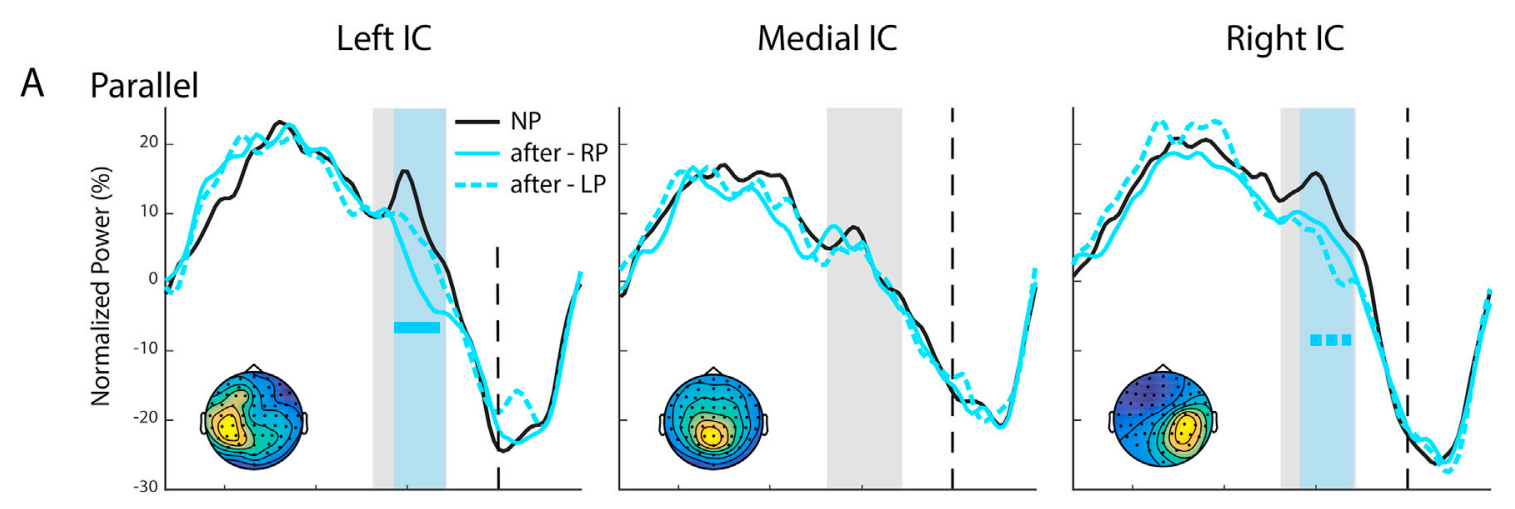

\section{B Cooperative}
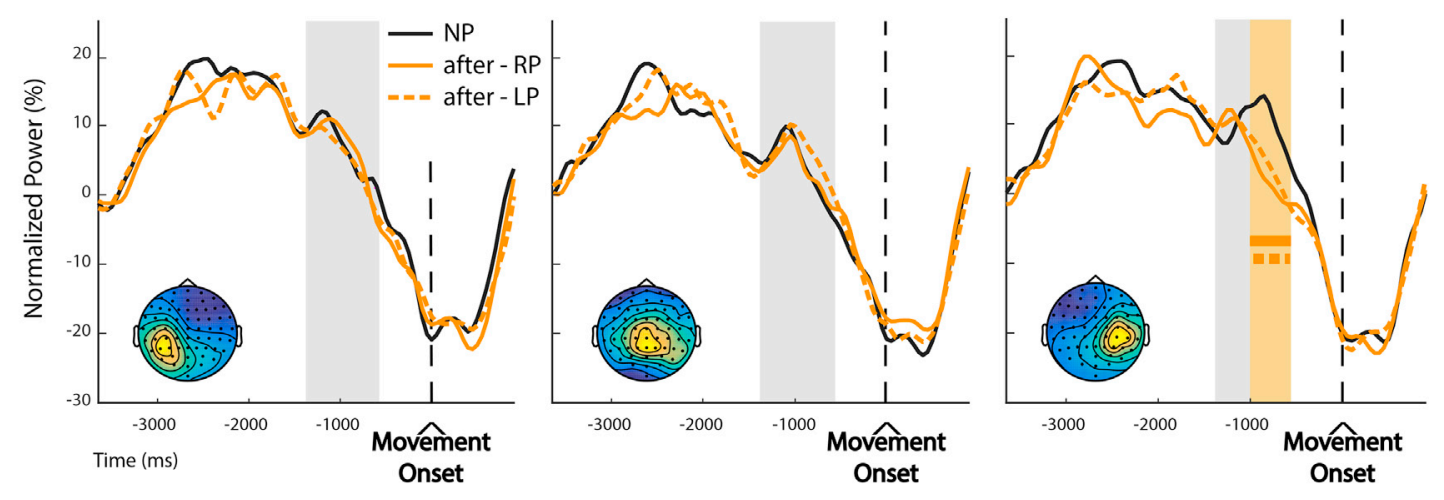

Fig. 8. Modulations of the foreperiod beta activity in the after-trials $n+1$ for the Parallel and Cooperative tasks.

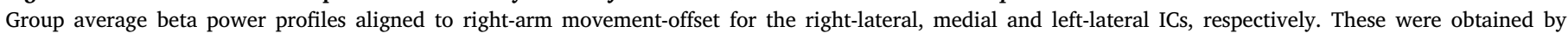

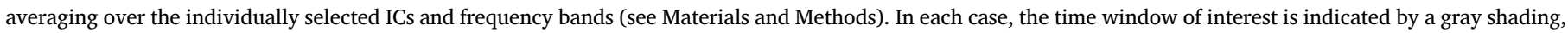

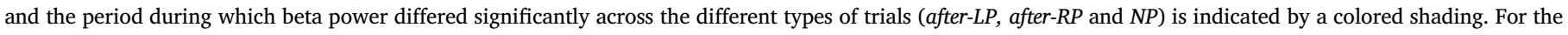

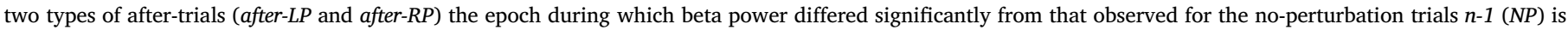

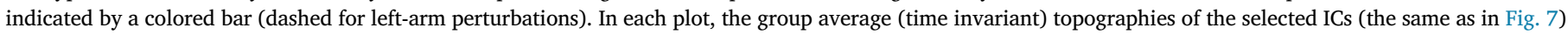
are presented.

factor. The periods over which kinematic-errors had significant effect on beta power are indicated for the Unimanual task by green bars (significant t-tests) and for the bimanual tasks by colored shadings (significant ANOVAs). For the bimanual tasks, at each significant time-point, we further proceeded to two post-hoc pairwise comparisons, contrasting each left- or right-arm perturbation ( $L P$ or $R P$ ) condition (trials $n$ ) to the no-perturbation baseline (NP) condition (trials $n-1$ ). In Fig. 6, the sampling points that survived the Bonferroni correction are indicated by colored bars.

For the Unimanual task (Fig. 5), t-tests revealed that the medial ICs exhibited significant sensitivity to the perturbations, applied in trials $n$, from $25 \mathrm{~ms}$ to the end of the tested time window. No significant effect was observed for the left ICs, whereas for the right IC beta power showed an error-related reduction lasting $200 \mathrm{~ms}$ (from 325 to $525 \mathrm{~ms}$ ). For both bimanual tasks, only the medial ICs showed significant error-related responses. In the Parallel task (Fig. 6A) repeated measures ANOVAs revealed that the power profiles of the medial ICs were significantly modulated during two epochs lasting respectively $150 \mathrm{~ms}$ (from 225 to $375 \mathrm{~ms}$ ) and $275 \mathrm{~ms}$ (from 725 to $1000 \mathrm{~ms}$ ). Pairwise $t$-test comparisons showed that both left-arm and right-arm perturbation significantly reduced beta power during about $200 \mathrm{~ms}$ (from 775 to $975 \mathrm{~ms}$ and from 825 to $1000 \mathrm{~ms}$, respectively). In the Cooperative task (Fig. 6B), a significant perturbation effect for the medial IC lasting $450 \mathrm{~ms}$ (from 375 to $825 \mathrm{~ms}$ ) was revealed by repeated measures ANOVAs. Pairwise $t$-test comparisons showed that a significant beta power reduction was produced when the perturbation was applied to the left arm, lasting $400 \mathrm{~ms}$ (from 325 to $725 \mathrm{~ms}$ ), whereas the right-arm perturbations induced a reduction over $300 \mathrm{~ms}$ (from 575 to $875 \mathrm{~ms}$ ).

We also proceeded to comparisons across tasks, in which we considered the beta power profiles observed in the baseline noperturbation $(N P)$ condition. For each type of ICs (medial, left- and right-lateral ICs), we conducted repeated measures ANOVAs with the task (Unimanual, Parallel and Cooperative) as within-subject factor. We found no significant differences between the amplitudes of the beta rebounds observed for the different tasks, even though a slightly larger beta rebound was observed for the Unimanual task than for the bimanual tasks.

Still considering the baseline no-perturbation (NP) trials, for each task we contrasted beta power across the different ICs (medial, left- and rightlateral ICs). For the Unimanual task, the point by point repeated measures ANOVAs revealed that beta power differed significantly across ICs (from 75 to 825 ms relative to movement offset). Pairwise tests indicated that the right-lateral ICs exhibited weaker post-movement synchronizations than the medial ICs (from 525 to $675 \mathrm{~ms}$ ) and the left-lateral ICs (from 75 to $275 \mathrm{~ms}$ ). For the Parallel task, beta power differed significantly across ICs (from 575 to $1000 \mathrm{~ms}$ relative to movement offset). Pairwise tests indicated that the right-lateral ICs exhibited weaker post-movement synchronizations than the medial ICs (from 975 to $1000 \mathrm{~ms}$ ) and the left-lateral ICs (from 575 to $725 \mathrm{~ms}$ ). No significant difference was found for the Cooperative task.

In the participants (11/15) for which several medial ICs exhibited a clear beta rebound, the most posterior of the latter showed the largest 
beta rebound and was thus selected. However, as complementary tests for these participants, we also analyzed the beta activities of the ICs with largest weighting at FCz-Fz. For these ICs, in the Unimanual task, beta power was significantly decreased during $250 \mathrm{~ms}$. However, for the Parallel and Cooperative tasks no significant beta rebound reduction was observed.

Foreperiod beta activity. For all ICs, around $1000 \mathrm{~ms}$ before movement onset a local peak was visible (Figs. 7-8), consistent with our previous findings (Torrecillos et al., 2015). To test whether the foreperiod beta activity in trials $n+1$ was influenced by the reach errors experienced in trials $n$, at each time point within the foreperiod-window of interest (see Material and Methods, indicated by gray shading), we computed a $t$-test (Unimanual task) or a repeated-measures ANOVAs (Parallel and Cooperative tasks) with the condition (after-LP, after-RP and $N P$ ) as within-subject factor. The periods where the main effect of condition was significant are indicated by colored shadings. For the bimanual tasks, at each significant time point, we further proceeded to two post-hoc pairwise comparisons, contrasting the trials $n+1$ following a left-arm or a right-arm perturbation (after-LP or after-RP) to the no-perturbation (NP) trials $n$-1. In each case, in Fig. 8 the sampling points that survived the Bonferroni correction are indicated by a thick line.

We first analyzed the pre-movement beta power time-courses of the different ICs (medial, left- and right-lateral ICs) for each reaching task separately. In all cases, we found no significant error-related response for the medial ICs. In contrast, significant effects of experienced kinematicerrors were found for the lateral ICs. For the Unimanual task (Fig. 7), the beta power profiles of both the left and right ICs presented significant beta power reductions. The beta power reduction was observed earlier and lasted longer for the left IC contralateral to the moving arm ( $400 \mathrm{~ms}$; from -1275 to $-875 \mathrm{~ms}$ relative to movement onset) than for the right IC ( $175 \mathrm{~ms}$; from -825 to $-650 \mathrm{~ms}$ ). For the Parallel task (Fig. 8A), beta activities of both lateral ICs were significantly modulated in response to the perturbations. Significant effects lasted $425 \mathrm{~ms}$ ( -1075 to $-650 \mathrm{~ms})$ and $400 \mathrm{~ms}$ ( -1075 to $-675 \mathrm{~ms}$ ), for the left- and the right-lateral ICs, respectively. In addition, post-hoc comparisons revealed symmetrical patterns for the two lateral ICs. For the left ICs the transient beta power enhancement was significantly attenuated when the right contralateral arm was perturbed ( $400 \mathrm{~ms} ;-1075$ to $-675 \mathrm{~ms}$ ), whereas the attenuations observed for left-arm perturbations did not reach significance. In the opposite way, the right ICs showed significant beta power reduction upon left-arm perturbations, while perturbations of the ipsilateral right arm induced reductions that remained statistically not significant (Fig. 8A). A clearly different pattern of oscillatory responses was observed in the Cooperative task (Fig. 8B). In this task, only the right ICs exhibited sensitivity to reach errors, irrespective of the arm on which the perturbations were applied; significant beta power reductions were observed, lasting respectively $300 \mathrm{~ms}$ ( -925 to $-650 \mathrm{~ms}$ ) and $325 \mathrm{~ms}$ ( -975 to $-650 \mathrm{~ms}$ ) for left-arm and right-arm perturbations.

We also compared across tasks the beta power profiles observed in the no-perturbation $(N P)$ condition. For each of the different ICs, we ran repeated measures ANOVAs at each time point within the predefined premovement-window of interest with the task (Unimanual, Parallel and Cooperative) as within-subject factor. No significant differences were found.

Dipole fitting and Measure Projection Analysis (MPA) of the ICs. For each task and each participant, we computed the equivalent current dipole model for the different ICs, medial and lateral (left and right). Fig. 9A shows the individual dipole solutions for the individual ICs together with the corresponding group-averaged topographies. The domains identified by MPA are represented in Fig. 9B, and Table 1 lists, for each task, the anatomical locations associated with each ERSP domain based on the Laboratory of NeuroImaging (LONI) project probabilistic atlas (Shattuck et al., 2008).

To summarize, the present results demonstrate several functional

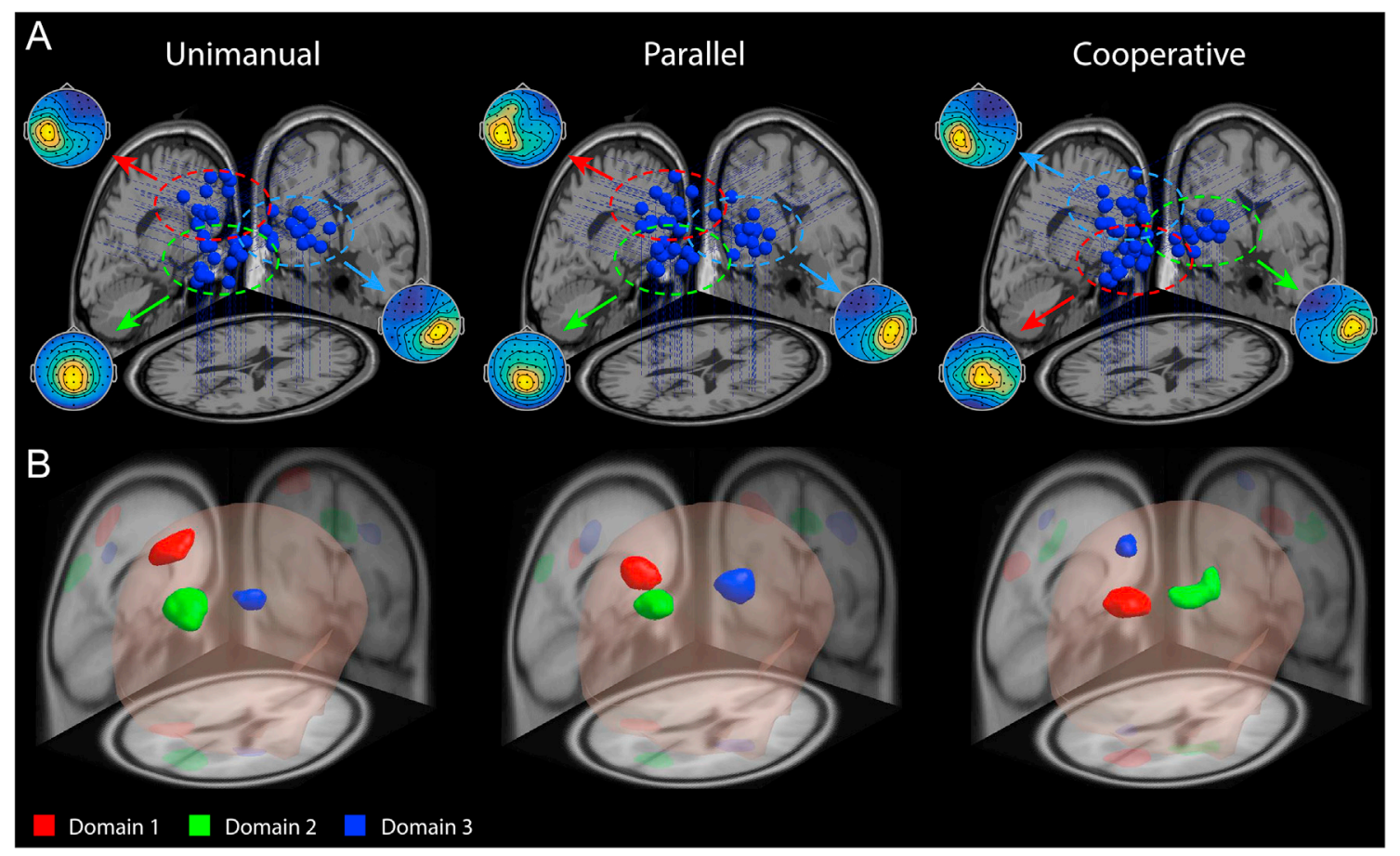

Fig. 9. IC dipoles and MPA domains for the Unimanual, Parallel and Cooperative tasks.

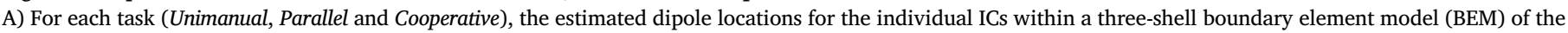

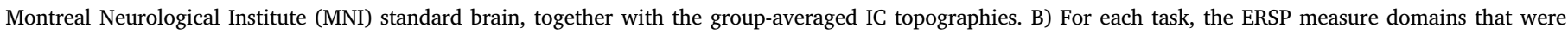

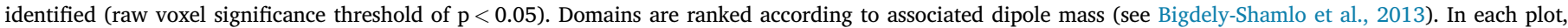

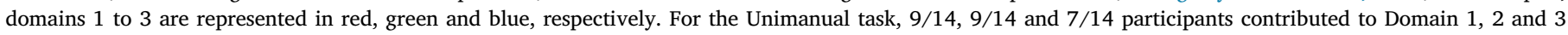

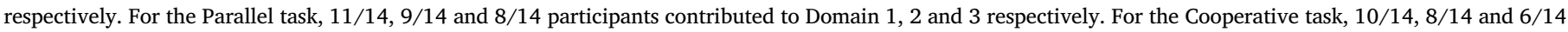
participants contributed to Domain 1,2 and 3 respectively. 
Table 1

Anatomical areas associated with the ERSP domains.

\begin{tabular}{|c|c|c|c|}
\hline Task & Domain & Anatomical Areas & Probability \\
\hline \multirow[t]{9}{*}{ Unimanual } & Domain 1 & L Superior Parietal Gyrus & 0.94 \\
\hline & \multirow[t]{6}{*}{ Domain 2} & $\mathrm{R}$ Precuneus & 0.37 \\
\hline & & R Cuneus & 0.19 \\
\hline & & L Precuneus & 0.19 \\
\hline & & R Superior Occipital Gyrus & 0.10 \\
\hline & & R Superior Parietal Gyrus & 0.06 \\
\hline & & L Cuneus & 0.06 \\
\hline & \multirow[t]{2}{*}{ Domain 3} & R Superior Parietal Gyrus & 0.83 \\
\hline & & R Angular Gyrus & 0.14 \\
\hline \multirow[t]{12}{*}{ Parallel } & \multirow[t]{4}{*}{ Domain 1} & L Superior Parietal Gyrus & 0.52 \\
\hline & & L Supramarginal Gyrus & 0.22 \\
\hline & & L Postcentral Gyrus & 0.19 \\
\hline & & L Angular Gyrus & 0.06 \\
\hline & \multirow[t]{4}{*}{ Domain 2} & $\mathrm{R}$ Precuneus & 0.53 \\
\hline & & R Cuneus & 0.19 \\
\hline & & L Precuneus & 0.11 \\
\hline & & R Superior Parietal Gyrus & 0.06 \\
\hline & \multirow[t]{4}{*}{ Domain 3} & R Postcentral Gyrus & 0.74 \\
\hline & & R Precentral Gyrus & 0.13 \\
\hline & & R Superior Parietal Gyrus & 0.07 \\
\hline & & R Supramarginal Gyrus & 0.05 \\
\hline \multirow[t]{10}{*}{ Cooperative } & \multirow[t]{4}{*}{ Domain 1} & $\mathrm{R}$ Precuneus & 0.64 \\
\hline & & L Precuneus & 0.13 \\
\hline & & R Cuneus & 0.09 \\
\hline & & R Superior Parietal Gyrus & 0.08 \\
\hline & \multirow[t]{4}{*}{ Domain 2} & R Postcentral Gyrus & 0.55 \\
\hline & & R Superior Parietal Gyrus & 0.25 \\
\hline & & R Supramarginal Gyrus & 0.11 \\
\hline & & R Precentral Gyrus & 0.07 \\
\hline & \multirow[t]{2}{*}{ Domain 3} & L Superior Parietal Gyrus & 0.85 \\
\hline & & L Postcentral Gyrus & 0.15 \\
\hline
\end{tabular}

differences between the foreperiod and post-movement beta activities. In addition, they suggest distinct neuronal substrates. While kinematic errors in trials $n$ attenuated the post-movement beta rebound of the medial IC, no significant modulation was observed for the lateral ICs during the same time window. Conversely, activities of the lateral ICs were significantly modulated during the preparation of the movements $n+1$ that directly followed a trial in which a kinematic error was experienced, whereas the activities of the medial ICs were not altered. Moreover, the current observations reveal that the foreperiod and post-movement beta activities are differently modulated by reach-error depending on the nature of the interlimb coordination. The medial ICs exhibited an attenuation of the post-movement beta rebound in all tasks, whichever arm was perturbed. In contrast, during movement preparation, the activities of the lateral ICs were differently modulated according to the interlimb coordination that was involved. In the Parallel task, the short beta power enhancement of the left IC was the most attenuated when the right contralateral arm was perturbed, whereas the right IC showed the largest response when the left arm was perturbed. A clearly different pattern was observed for the Cooperative task, in which only beta power of the right IC exhibited an error-related attenuation, regardless of the arm to which the perturbation was applied.

Note that, in contrast to previous studies using MPA (Ofori et al., 2015), here we used MPA (Measure Projection Analysis) only to provide additional support for the idea that distinct neural structures underlie the foreperiod and the post-movement beta-power error-related modulations; consistent with this hypothesis, distinct domains were obtained from the ERSP of the medial, left- and right-lateral ICs. As a consequence, the presentation of our results does not follow the report structure of previous studies using MPA (e.g. Ofori et al., 2015). In those studies, based on an "omnibus" ERSP analysis, the MPA domains are first defined. Then, in a second step, different statistical tests of interest are run on the ERSP of the ICs contributing to the different domains (e.g. contrasts between ERSP patterns associated with different condition-trials).

In contrast, here we first analyzed the ERSP of the selected ICs, and then we found that the different types of ICs (medial, left- and right- lateral) contributed to different MPA domains. The latter MPA finding is critical, since it means that, here, the two approaches (performing ERSP statistical analyses before or after creating the MPA domains) are essentially equivalent. We chose a presentation format that facilitates the comparison of the current results with those reported in Torrecillos et al. (2015).

\section{Discussion}

Previous results have shown that the foreperiod and the postmovement beta activities present functional differences (Torrecillos et al., 2015). Here, we report two main new findings. First, exploiting ICA as a blind source-separation method, we found a double dissociation strongly suggesting that distinct neural structures are recruited in the error-related beta modulations observed before and after movement. On the one hand, the error-related beta rebound reduction was best captured by ICs characterized by medial topographies, which however did not account for the foreperiod modulation. On the other hand, lateral ICs captured the foreperiod responses, but not the post-movement beta rebound attenuation.

Second, contrasting bimanual tasks in which identical unilateral perturbations triggered different trial-to-trial motor-command updates, we demonstrate that the error-related modulation of the foreperiod beta activity does not reflect changes in the motor output from primary motor cortex, and suggest that it may instead relate to higher-level processing of sensory afferents, critical for sensorimotor adaptation.

\subsection{Post-movement beta rebound modulation was observed medially regardless of the mode of interlimb coordination}

In all reaching tasks (uni- or bimanual) the kinematic errors induced by the unilateral mechanical perturbations were associated with clear reductions of the beta rebound. Furthermore, in all cases (that is, regardless of whether movements were performed with both or one arm only) the post-movement beta power modulations were captured by ICs presenting medial topographies. At first glance, these medial topographies might appear at odds with the report by Tan et al. (2014) who uncovered this movement error-related response. These authors analyzed the beta rebound reduction for electrodes located over the sensorimotor cortex contralateral to the moving hand (C3-Cz), at which they recorded the largest beta rebound. We previously observed an analogue response but more weakly lateralized (Torrecillos et al. (2015). These differences in topographies may reflect somatotopical specificity (Salmelin et al., 1995; Neuper and Pfurtscheller, 1996; Brovelli et al., 2002). Tan et al. (2014) used a hand controlled joystick task, whereas in Torrecillos et al. (2015) participants performed whole upper-limb reaches, involving proximal control (elbow and shoulder). The results of our ICA (largest rebound for medial ICs) are in fact consistent with the slightly lateralized beta rebound recorded in sensor space for unimanual reaches. Indeed, the beta rebound was largest for the medial ICs, but it was also clearly represented in the lateral ICs. In the Unimanual task, it was significantly more pronounced for the left than for the right lateral ICs. Hence, mixing the different ICs results in a beta rebound slightly contralateral in sensor space.

However, it is important to mention that the cortical generators of the beta rebound remain uncertain. In fact, results converge on the idea that the beta rebound does not arise from a discrete cortical locus, but rather from a distributed cortical network (for a review, see Kilavik et al., 2013). Here, it is also important to keep in mind that the site of its maximum modulation might not coincide with the location of its maximum amplitude. In the present study, for most participants (11/15) several medial ICs could be identified exhibiting a clear beta rebound. In these cases, the IC with the largest weight over the post-central cortex showed the largest effects. Interestingly, in a recent study Fischer et al. (2016) reported post-movement beta power modulations over medio-parietal regions $(\mathrm{Pz})$. These authors examined beta activity during a rhythmic 
finger-tapping task in which participants had to stop abruptly or continue tapping in response to an auditory cue. Changes in beta power were linked to the reaction time in the trial following the cue, such that a greater decrease in beta power was associated with a larger increase in reaction time. These observations are consistent with the idea that the attenuation of the post-movement beta activity relates to an increase in alertness, which may be due to task demands (Fischer et al., 2016) or elicited by salient errors/events (Tan et al., 2014; Torrecillos et al., 2015). However, they do not allow determining whether post-movement beta activity relates to adaptive reorganizational processes or not, since error/mismatch-detection mechanisms can be recruited without driving adaptation (Diedrichsen et al., 2005; Torrecillos et al., 2015).

As expected, in both bimanual tasks the unilateral mechanical perturbations produced asymmetrical reach errors. In the perturbed catchtrials $(n)$, large deviations were observed for the perturbed arm, while no (in the Parallel task) or small (in the Cooperative task) deviations were observed for the non-perturbed arm. In the reaches $(n+1)$ performed immediately after an error was experienced, the "after-effects" presented corresponding interlimb asymmetries. However, these asymmetries were not reflected in the post-movement oscillatory responses. This agrees with the idea that the beta rebound attenuation may relate to error/ mismatch-detection mechanisms which can intervene independent of sensorimotor remapping (update of internal models). We put forward this hypothesis (Torrecillos et al., 2015) to explain that the beta rebound is reduced when a salient unexpected change in the environment perturbs the movement, even though it does not trigger adaptive behavioral adjustment in the following trial.

\subsection{Foreperiod beta-power modulation exhibited different interhemispheric asymmetries depending on the interlimb coordination}

When no perturbation was experienced in the preceding trial, beta power exhibited a local peak around $1 \mathrm{~s}$ before movement initiation, consistent with our previous findings (Torrecillos et al., 2015). This peak followed a large and long lasting enhancement, most likely induced by the passive movement (Cassim et al., 2001; Alegre et al., 2002; Müller et al., 2003) back to the start position imposed by the robot at the end of each trial. Even though visible in the medial ICs as well, this short increase in beta power was best captured by ICs presenting clearly lateralized topographies. Critically, it was only the latter ICs that accounted for its error-related reduction.

Here analyzing beta power over both hemispheres, in the Unimanual task we found that the foreperiod beta enhancement was reduced bilaterally, albeit more weakly and slightly later ispilaterally. The oscillatory responses elicited in the Parallel task presented analogue patterns. The foreperiod transient beta power enhancement was also reduced over both hemispheres, with a prominent contralateral reduction and a weaker modulation ipsilaterally, which in this case did not reach significance though. The ipsilateral foreperiod beta modulations that we observed in the Unimanual and Parallel tasks could reflect efferent signal modulations, which however would not translate into visible changes in the motor output. This idea would agree with classical electrophysiological (Tanji et al., 1988; Donchin et al., 2002; Ganguly et al., 2009) and more recent imaging (Diedrichsen et al., 2013; Wiestler and Diedrichsen, 2013; Waters et al., 2017) studies demonstrating that activity in the primary motor cortex contains information about the ongoing ipsilateral movements, which may contribute to movement control either through directly descending ipsilateral projections, or by shaping the activation patterns on the contralateral side.

However, in the Cooperative task a clear uncoupling between the trialto-trial behavioral updates and the electrophysiological responses was observed. After the unilateral perturbations, adaptive responses were visible for both arms, whereas significant beta power modulation was observed only over the right hemisphere regardless of the side of the perturbation. This finding is critical as it demonstrates that the foreperiod beta activity does not reflect efferent processes that shape the muscle activation pattern. While beta activity has been traditionally considered in relation to movement execution, there is growing evidence that sensorimotor beta oscillations should be interpreted within a framework encompassing both efferent and afferent signal processing (Van Ede and Maris, 2013; Palmer et al., 2016). The neural mechanisms of sensorimotor adaptation still remain uncertain, but it has been demonstrated that somatosensory areas play a key role (Krebs et al., 1998; Pavlides et al., 1993; Vidoni et al., 2010; Ostry and Gribble, 2016; Mathis et al., 2017). Thus, we may speculate that the beta modulation observed during the foreperiod may reflect mechanisms involved in the processing and integration of somatosensory information critical for the adaptive update of the upcoming movement. Clearly, the current results do not permit determining the exact nature of the processes that are reflected by the foreperiod beta modulation. Nonetheless, they provide two pieces of information that should guide present conjectures and future research.

First, they indicate that the involved processes are affected by the goal of the bimanual task, and thus the interlimb coordination mode. Several imaging studies have demonstrated that brain activation patterns during bimanual movements are task- and condition-dependent (Puttemans et al., 2005; Johansson et al., 2006; Theorin and Johansson, 2007; Grefkes et al., 2008; Duque et al., 2009; Goble et al., 2010). In a recent fMRI experiment, Dietz et al. (2015) directly contrasted a cooperative "bottle-opening" task with non-cooperative or isometric bimanual tasks. They found increased BOLD activation in the secondary (S2) and to a lesser degree in the primary (S1) somatosensory areas during the "bottle-opening" relative to the other tasks. Critically, while (as expected from numerous previous studies on non-cooperative movements) strong BOLD activation was found in the supplementary motor areas, the premotor and primary motor cortices for all bimanual tasks, none of these regions exhibited significant activation changes depending on the mode of interlimb coordination.

The second piece of information lies in the right-lateralized modulation that was observed in the Cooperative task. Recently, Le et al. (2014, 2016) used TMS to explore the neural underpinnings of goal-directed bimanual grasp and reach-to-grasp movements. They showed that transient disruption by TMS of the right anterior-lateral superior parietal lobule (BA7; considered to be concerned with the highest levels of somatosensory integration; Jones and Powell, 1970) affected bimanual reach-to-grasp actions in their transport and grip components (Le et al., 2016). According to the authors, these effects mirror the recruitment of a right lateralized 'integration network' essential for a comprehensive spatial representation of the body needed for bimanual actions (Corbetta et al., 2000; Marshall and Fink, 2001; Barthelemy and Boulinguez, 2001; Blanke et al., 2002; Wenderoth et al., 2004; Serrien et al., 2006; Weiss et al., 2006). Here, it is important to notice that Le et al. (2014, 2016) observed significant effects of TMS only in trials in which the movement was disrupted by a change of the object size ("size-perturbation" at movement onset) increasing the difficulty of the action, not in the unperturbed control trials. One may draw a parallel between the specificity of this effect and the findings by Rueda-Delgado et al. (2017). As evoked in the Introduction, these authors examined beta activity during a task in which participants had to draw a line using both hands, and in which they manipulated the level difficulty. Contrary to their hypothesis, they found that only right-lateralized sensorimotor regions exhibited modulation of beta power as a function of task difficulty. They explain their unexpected results as follows. While the left hemisphere would exert primary control over hand movements, including bimanual movements (for a review, see Serrien et al., 2006), additional neural resources would be recruited from the right hemisphere in challenging situations. In our experiments, challenges arose from the application of mechanical perturbations. Thus, the right-lateralized pattern that we observed in the Cooperative task seems to converge with the results of these previous studies.

\subsection{Conclusions and open issues}

Contrasting error-related beta power modulations in different 
reaching tasks, we did not find reflection of the task difference for the beta rebound, whereas the foreperiod beta activity was clearly differently modulated depending on the mode of interlimb coordination. These observations fit with the idea that the attenuation of the beta rebound may relate to salient error/mismatch detection mechanisms, independent of implicit sensorimotor remapping (internal model update), while in contrast the foreperiod beta modulation may reflect adaptive processes. Indeed our idea was that beta activity reflecting sensorimotor adaptation should be influenced by the interlimb coordination, as are the behavioral adaptive responses.

Previous results limited to unimanual movements (Torrecillos et al., 2015) were compatible with the idea that the foreperiod beta modulation relates to changes in the upcoming motor output, fitting with the proposition that attenuations of the beta power facilitate changes in the motor set (Engel and Fries, 2010). In contrast, the complex picture offered by the present findings rules out this functional interpretation. The uncoupling between trial-to-trial motor-command update and error-related foreperiod beta modulation that we observed in the Cooperative task demonstrates that the foreperiod beta activity does not mirror changes in the efferent signals, and may instead reflect high-level sensory integration processes involved in sensorimotor adaptation. However, the neural correlates of bimanual actions in which both hands cooperate have been remained largely unexplored so far, and even the interhemispheric interaction involved in unimanual movements still need to be clarified. As a consequence, the present results do not permit determining the exact nature of the processes reflected by the foreperiod beta activity. Yet, by extending the analysis of error-related beta oscillations to new contexts, at the risk of yielding more questions than answers, the current study provide directions for future research.

\section{Acknowledgments}

This research was supported by the European Union H2020 - Marie Sklodowska-Curie 2014-2015 - ITN/ETN - GA n ${ }^{\circ} 642961$ PACE program. We thank Bill Mackay, Jean Blouin, Thomas Brochier and Liana Brown for helpful comments and suggestions. We also thank Juliana Gonzalez Astudillo for the programming of the robot. The authors declare no competing financial interest.

\section{References}

Aguera, P.E., Jerbi, K., Caclin, A., Bertrand, O., 2011. Elan: a software package for analysis and visualization of MEG, EEG, and LFP signals. Comput. Intell. Neurosci. 2011, 158970.

Alegre, M., Labarga, A., Gurtubay, I.G., Iriarte, J., Malanda, M., Artieda, J., 2002. Beta electroencephalograph changes during passive movements: sensory afferences contribute to beta event-related desynchronization in humans. Neurosci. Lett. 331, 29-32.

Alegre, M., de Gurtubay, I.G., Labarga, A., Iriarte, J., Malanda, A., Artieda, J., 2004. Alpha and beta oscillatory activity during a sequence of two movements. Clin. Neurophysiol. 115, 124-130.

Alegre, M., Imirizaldu, L., Valencia, M., Iriarte, J., Arcocha, J., Artieda, J., 2006. Alpha and beta changes in cortical oscillatory activity in a go/no go randomly-delayedresponse choice reaction time paradigm. Clin. Neurophysiol. 117, 16-25.

Barthelemy, S., Boulinguez, P., 2001. Manual reaction time asymmetries in human subjects: the role of movement planning and attention. Neurosci. Lett. 315, 41-44.

Bigdely-Shamlo, N., Mullen, T., Kreutz-Delgado, K., Makeig, S., 2013. Measure projection analysis: a probabilistic approach to EEG source comparison and multi- subject inference. Neuroimage 72, 287-303.

Blanke, O., Ortigue, S., Landis, T., Seeck, M., 2002. Stimulating illusory own-body perceptions. Nature 419, 269-270.

Brovelli, A., Battaglini, P.P., Naranjo, J.R., Budai, R., 2002. Medium-range oscillatory network and the 20-Hz sensorimotor induced potential. Neuroimage 16, 130-141.

Brovelli, A., Ding, M., Ledberg, A., Chen, Y., Nakamura, R., Bressler, S.L., 2004. Beta oscillations in a large-scale sensorimotor cortical network: directional influences revealed by Granger causality. Proc. Natl. Acad. Sci. U.S.A. 101, 9849-9854.

Cassim, F., Monaca, C., Szurhaj, W., Bourriez, J.L., Defebvre, L., Derambure, P., Guieu, J.D., 2001. Does post-movement beta synchronization reflect an idling motor cortex? Neuroreport 12, 3859-3863.

Cheyne, D., Gaetz, W., Garnero, L., Lachaux, J.P., Ducorps, A., Schwartz, D., Varela, F.J., 2003. Neuromagnetic imaging of cortical oscillations accompanying tactile stimulation. Cognit. Brain Res. 17, 599-611.
Courtemanche, R., Fujii, N., Graybiel, A.M., 2003. Synchronous, focally modulated betaband oscillations characterize local field potential activity in the striatum of awake behaving monkeys. J. Neurosci. 23, 11741-11752.

Courtemanche, R., Lamarre, Y., 2005. Local field potential oscillations in primate cerebellar cortex: synchronization with cerebral cortex during active and passive expectancy. J. Neurophysiol. 93, 2039-2052.

Corbetta, M., Kincade, J.M., Ollinger, J.M., McAvoy, M.P., Shulman, G.L., 2000. Voluntary orienting is dissociated from target detection in human posterior parietal cortex. Nat. Neurosci. 3, 292-297.

Crone, N.E., Miglioretti, D.L., Gordon, B., Sieracki, J.M., Wilson, M.T., Uematsu, S., Lesser, R.P., 1998. Functional mapping of human sensorimotor cortex with electrocorticographic spectral analysis. I. Alpha and beta event-related desynchronization. Brain 121, 2271-2299.

Delorme, A., Palmer, J., Onton, J., Oostenveld, R., Makeig, S., 2012. Independent EEG sources are dipolar. PLoS One 7, e30135. https://doi.org/10.1371/journal.pone. 0030135.

Delorme, A., Makeig, S., 2004. EEGLAB: an open source toolbox for analysis of single-trial EEG dynamics including independent component analysis. J. Neurosci. Meth. 134, 9-21.

Denker, M., Roux, S., Lindén, H., Diesmann, M., Riehle, A., Grün, S., 2011. The local field potential reflects surplus spike synchrony. Cerebr. Cortex 21, 2681-2695.

Diedrichsen, J., Wiestler, T., Krakauer, J.W., 2013. Two distinct ipsilateral cortical representations for individuated finger movements. Cerebr. Cortex 23, 1362-1377.

Diedrichsen, J., Hashambhoy, Y., Rane, T., Shadmehr, R., 2005. Neural correlates of reach errors. J. Neurosci. 25, 9919-9931.

Diedrichsen, J., 2007. Optimal task-dependent changes of bimanual feedback control and adaptation. Curr. Biol. 17, 1675-1679.

Dietz, V., Macauda, G., Schrafl-Altermatt, M., Wirz, M., Kloter, E., Michels, L., 2015. Neural coupling of cooperative hand movements: a reflex and fMRI study. Cerebr. Cortex 25, 948-958.

Donchin, O., Gribova, A., Steinberg, O., Mitz, A.R., Bergman, H., Vaadia, E., 2002. Singleunit activity related to bimanual arm movements in the primary and supplementary motor cortices. J. Neurophysiol. 88, 3498-34517.

Donchin, O., Gribova, A., Steinberg, O., Bergman, H., Vaadia, E., 1998. Primary motor cortex is involved in bimanual coordination. Nature 395, 274-278.

Duque, J., Davare, M., Delaunay, L., Jacob, B., Saur, R., Hummel, F., Hermoye, L., Rossion, B., Olivier, E., 2009. Monitoring coordination during bimanual movements: where is the mastermind? J. Cognit. Neurosci. 22, 526-542.

Engel, A.K., Fries, P., 2010. Beta-band oscillations — signalling the status quo? Curr Opin. Neurobiol. 20, 156-165.

Fischer, T., Langner, R., Diers, K., Brocke, B., Birbaumer, N., 2010. Temporo-spatial dynamics of event-related EEG beta activity during the initial contingent negative variation. PLoS One 5, e12514.

Fischer, P., Tan, H., Pogosyan, A., Brown, P., 2016. High post-movement parietal low-beta power during rhythmic tapping facilitates performance in a stop task. Eur. J. Neurosci. 44, 2202-2213.

Fowler, B., Duck, T., Mosher, M., Mathieson, B., 1991. The coordination of bimanual aiming movements: evidence for progressive desynchronization. Q. J. Exp. Psychol. 43, 205-221.

Ganguly, K., Secundo, L., Ranade, G., Orsborn, A., Chang, E.F., Dimitrov, D.F. Wallis, J.D., Barbaro, N.M., Knight, R.T., Carmena, J.M., 2009. Cortical representation of ipsilateral arm movements in monkey and man. J. Neurosci. 29, 12948-12956.

Goble, D.J., Coxon, J.P., Van Impe, A., De Vos, J., Wenderoth, N., Swinnen, S.P., 2010. The neural control of bimanual movements in the elderly: brain regions exhibiting age-related increases in activity, frequency-induced neural modulation, and taskspecific compensatory recruitment. Hum. Brain Mapp. 31, 1281-1295.

Grefkes, C., Eickhoff, S.B., Nowak, D.A., Dafotakis, M., Fink, G.R., 2008. Dynamic intraand interhemispheric interactions during unilateral and bilateral hand movements assessed with fMRI and DCM. Neuroimage 41, 1382-1394.

Johansson, R.S., Theorin, A., Westling, G., Andersson, M., Ohki, Y., Nyberg, L., 2006. How a lateralized brain supports symmetrical bimanual tasks. PLoS Biol. 4, e158.

Jones, E.G., Powell, T.P., 1970. An anatomical study of converging sensory pathways within the cerebral cortex of the monkey. Brain 93, 793-820.

Kelso, J.A., Southard, D.L., Goodman, D., 1979. On the nature of human interlimb coordination. Science 203, 1029-1031 (New York, NY).

Kilavik, B.E., Zaepffel, M., Brovelli, A., MacKay, W.A., Riehle, A., 2013. The ups and downs of beta oscillations in sensorimotor cortex. Exp. Neurol. 245, 15-26.

Krebs, H.I., Brashers-Krug, T., Rauch, S.L., Savage, C.R., Hogan, N., Rubin, R.H., Fischman, A.J., Alpert, N.M., 1998. Robot-aided functional imaging: application to a motor learning study. Hum. Brain Mapp. 6, 59-72.

Le, A., Vesia, M., Yan, X., Niemeier, M., Crawford, J.D., 2014. The right anterior intraparietal sulcus is critical for bimanual grasping: a TMS study. Cerebr. Cortex 24, 2591-2603.

Le, A., Vesia, M., Yan, X., Crawford, J.D., Niemeier, M., 2016. Parietal area BA7 integrates motor programs for reaching, grasping, and bimanual coordination. J. Neurophysiol. $117,624-636$.

Lebar, N., Danna, J., Moré, S., Mouchnino, L., Blouin, J., 2017. On the neural basis of sensory weighting: alpha, beta and gamma modulations during complex movements. Neuroimage 150, 200-212.

Liuzzi, G., Horniss, V., Zimerman, M., Gerloff, C., Hummel, F.C., 2011. Coordination of uncoupled bimanual movements by strictly timed interhemispheric connectivity. J. Neurosci. 31, 9111-9117.

Makeig, S., Jung, T.P., Bell, A.J., Ghahremani, D., Sejnowski, T.J., 1997. Blind separation of auditory event related brain responses into independent components. Proc. Natl. Acad. Sci. U.S.A. 94, 10979-10984. 
Marshall, J.C., Fink, G.R., 2001. Spatial cognition: where we were and were we are. Neuroimage 14 (S2-S7), 765.

Marteniuk, R.G., MacKenzie, C.L., Baba, D.M., 1984. Bimanual movement control: information processing and interaction effects. Q. J. Exp. Psychol. 36A, 335-365.

Mathis, M.W., Mathis, A., Uchida, N., 2017. Somatosensory cortex plays an essential role in forelimb motor adaptation in mice. Neuron 93, 1493-1503.

McCombe Waller, S., Whitall, J., 2008. Bilateral arm training: why and who benefits? NeuroRehabilitation 23, 29-41.

Molnár, M., Csuhaj, R., Gaál, Z.A., Czigler, B., Ulbert, I., Boha, R., Kondákor, I., 2008 Spectral characteristics and linear-nonlinear synchronization changes of different EEG frequency bands during the CNV. Psychophysiology 45, 412-419.

Müller, G.R., Neuper, C., Rupp, R., Keinrath, C., Gerner, H.J., Pfurtscheller, G., 2003. Event-related beta EEG changes during wrist movements induced by functional electrical stimulation of forearm muscles in man. Neurosci. Lett. 340, 143-147.

Murthy, V.N., Fetz, E.E., 1996. Oscillatory activity in sensorimotor cortex of awake monkeys: synchronization of local field potentials and relation to behavior. J. Neurophysiol. 76, 3949-3967.

Murthy, V.N., Fetz, E.E., 1992. Coherent 25- to 35-Hz oscillations in the sensorimotor cortex of behaving monkeys. Proc. Natl. Acad. Sci. U.S.A. 89, 5670-5674.

Nagamine, T., Kajola, M., Salmelin, R., Shibasaki, H., Hari, R., 1996. Movement related slow cortical magnetic fields and changes of spontaneous MEG- and EEG-brain rhythms. Electroencephalogr. Clin. Neurophysiol. 199, 274-286.

Nauhaus, I., Busse, L., Carandini, M., Ringach, D.L., 2009. Stimulus contrast modulates functional connectivity in visual cortex. Nat. Neurosci. 12, 70-76.

Neuper, C., Pfurtscheller, G., 1996. Post-movement synchronization of beta rhythms in the EEG over the cortical foot area in man. Neurosci. Lett. 216, 17-20.

Obhi, S.S., 2004. Bimanual coordination: an unbalanced field of research. Mot. Contr. 8 , $111-120$.

Ofori, E., Coombes, S.A., Vaillancourt, D.E., 2015. 3D Cortical electrophysiology of ballistic upper limb movement in humans. Neuroimage 115, 30-41.

Oostenveld, R., Fries, P., Maris, E., Schoffelen, J.M., 2011. FieldTrip: open source software for advanced analysis of MEG, EEG and invasive electrophysiological data. Comput. Intell. Neurosci. 2011, 156869.

Oldfield, R.C., 1971. The assessment and analysis of handedness: the Edinburgh inventory. Neuropsychologia 9, 97-113.

Ostry, D.J., Gribble, P.L., 2016. Sensory plasticity in human motor learning. Trends Neurosci. 39 (2), 114-123.

Palmer, C., Zapparoli, L., Kilner, J.M., 2016. A new framework to explain sensorimotor beta oscillations. Trends Cognit. Sci. 20, 321-323.

Paradiso, G., Cunic, D., Saint-Cyr, J.A., Hoque, T., Lozano, A.M., Lang, A.E., Chen, R., 2004. Involvement of human thalamus in the preparation of self-paced movement. Brain 127, 2717-2731.

Pavlides, C., Miyashita, E., Asanuma, H., 1993. Projection from the sensory to the motor cortex is important in learning motor skills in the monkey. J. Neurophysiol. 70, 733-741.

Pfurtscheller, G., Lopes da Silva, F.H., 1999. Event-related EEG/MEG synchronization and desynchronization: basic principles. Clin. Neurophysiol. 110, 1842-1857.

Puttemans, V., Wenderoth, N., Swinnen, S.P., 2005. Changes in brain activation during the acquisition of a multifrequency bimanual coordination task: from the cognitive stage to advanced levels of automaticity. J. Neurosci. 25, 4270-4278.

Roelfsema, P.R., Engel, A.K., König, P., Singer, W., 1997. Visuomotor integration is associated with zero time-lag synchronization among cortical areas. Nature 385, $157-161$.

Rueda-Delgado, L.M., Solesio-Jofre, E., Mantini, D., Dupont, P., Daffertshofer, A., Swinnen, S.P., 2017. Coordinative task difficulty and behavioural errors are associated with increased long-range beta band synchronization. Neuroimage 146, 883-893.

Salmelin, R., Hämäläinen, M., Kajola, M., Hari, R., 1995. Functional segregation of movement-related rhythmic activity in the human brain. Neuroimage 2, 237-243.

Serrien, D.J., Ivry, R.B., Swinnen, S.P., 2006. Dynamics of hemispheric specialization and integration in the context of motor control. Nat. Rev. Neurosci. 7, 160-166.
Shattuck, D.W., Mirza, M., Adisetiyo, V., Hojatkashani, C., Salamon, G., Narr, K.L., Poldrack, R.A., Bilder, R.M., Toga, A.W., 2008. Construction of a 3D probabilistic atlas of human cortical structures. Neuroimage 39, 1064-1080.

Swinnen, S.P., 2002. Intermanual coordination: from behavioural principles to neuralnetwork interactions. Nat. Rev. Neurosci. 3, 348-359.

Tan, H., Jenkison, N., Brown, P., 2014. Dynamic neural correlates of motor error monitoring and adaptation during trial-to-trial learning. J. Neurosci. 34, 5678-5688.

Tan, H., Wade, C., Brown, P., 2016. Post-movement beta activity in sensorimotor cortex indexes confidence in the estimations from internal models. J. Neurosci. 36, 1516-1528.

Taniguchi, M., Kato, A., Fujita, N., Hirata, M., Tanaka, H., Kihara, T., Ninomiya, H., Hirabuki, N., Nakamura, H., Robinson, S.E., Cheyne, D., Yoshimine, T., 2000. Movement-related desynchronization of the cerebral cortex studied with spatially filtered magnetoencephalography. Neuroimage 12, 298-306.

Tanji, J., Okano, K., Sato, K., 1988. Neuronal activity in cortical motor areas related to ipsilateral, contralateral, bilateral digit movements of the monkey. J. Neurophysiol. 60, 325-343.

Theorin, A., Johansson, R.S., 2007. Zones of bimanual and unimanual preference within human primary sensorimotor cortex during object manipulation. Neuroimage 36 (Suppl. 2), T2-T15.

Thoroughman, K.A., Shadmehr, R., 2000. Learning of action through adaptive combination of motor primitives. Nature 407, 742-747.

Torrecillos, F., Albouy, P., Brochier, T., Malfait, N., 2014. Does the processing of sensory and reward-prediction errors involve common neural resources? Evidence from a frontocentral negative potential modulated by movement execution errors. J. Neurosci. 34, 4845-4856.

Torrecillos, F., Alayrangues, J., Kilavik, B.E., Malfait, N., 2015. Distinct modulations in sensorimotor postmovement and foreperiod beta-band activities related to error salience processing and sensorimotor adaptation. J. Neurosci. 35, 12753-12765.

Van Ede, F., Jensen, O., Maris, E., 2010. Tactile expectation modulates prestimulus beta -band oscillations in human sensorimotor cortex. Neuroimage 51, 867-876.

Van Ede, F., De Lange, F., Jensen, O., Maris, E., 2011. Orienting attention to an upcoming tactile event involves a spatially and temporally specific modulation of sensorimotor alpha- and beta-band oscillations. J. Neurosci. 31, 2016-2024.

Van Ede, F., Köster, M., Maris, E., 2012. Beyond establishing involvement, quantifying the contribution of anticipatory alpha- and beta-band suppression to perceptual improvement with attention. J. Neurophysiol. 108, 2352-2362.

Van Ede, F., Maris, E., 2013. Somatosensory demands modulate muscular Beta oscillations, independent of motor demands. J. Neurosci. 33, 10849-10857.

Van Wijk, B.C.M., Daffertshofer, A., Roach, N., Praamstra, P., 2009. A role of beta oscillatory synchrony in biasing response competition? Cerebr. Cortex 19, 1294-1302.

Vidoni, E.D., Acerra, N.E., Dao, E., Meehan, S.K., Boyd, L.A., 2010. Role of the primary somatosensory cortex in motor learning: an rTMS study. Neurobiol. Learn. Mem. 93, 532-539.

Waters, S., Wiestler, T., Diedrichsen, J., 2017. Cooperation not competition: bihemispheric tDCS and fMRI show role for ipsilateral hemisphere in motor learning. J Neurosci 3414-3416. https://doi.org/10.1523/JNEUROSCI.3414-16.2017 [Epub ahead of print].

Weiss, P.H., Rahbari, N.N., Lux, S., Pietrzyk, U., Noth, J., Fink, G.R., 2006. Processing the spatial configuration of complex actions involves right posterior parietal cortex: an fMRI study with clinical implications. Hum. Brain Mapp. 27, 1004-1014.

Wenderoth, N., Debaere, F., Sunaert, S., Van Hecke, P., Swinnen, S.P., 2004. Parietopremotor areas mediate directional interference during bimanual movements. Cerebr. Cortex 14, 1153-1163.

Wiestler, T., Diedrichsen, J., 2013. Skill learning strengthens cortical representations of motor sequences. Elife 9 (2), e00801. https://doi.org/10.7554/eLife.00801.

Witham, C.L., Wang, M., Baker, S.N., 2007. Cells in somatosensory areas show synchrony with beta oscillations in monkey motor cortex. Eur. J. Neurosci. 26, 2677-2686.

Zaepffel, M., Trachel, R., Kilavik, B.E., Brochier, T., 2013. Modulations of EEG beta power during planning and execution of grasping movements. PLoS One 8, e60060. 